\title{
Identification of a hypoxia-associated long non- coding RNA signature and nomogram as a prognostic target for clear cell renal cell carcinoma
}

Hualin Chen

First Affiliated Hospital of Chongqing Medical University

Yang Pan

First Affiliated Hospital of Chongqing Medical University

Xiaoxiang Jin

First Affiliated Hospital of Chongqing Medical University

Gang Chen ( $\nabla$ chengang2308@163.com )

First Affiliated Hospital of Chongqing Medical University

\section{Research Article}

Keywords: clear cell renal cell carcinoma, hypoxia, IncRNA signature, nomogram, TCGA

Posted Date: December 15th, 2020

DOI: https://doi.org/10.21203/rs.3.rs-126309/v1

License: (a) (i) This work is licensed under a Creative Commons Attribution 4.0 International License. Read Full License 


\section{Abstract \\ Background}

To develop a hypoxia-associated long non-coding (IncRNA) signature for risk stratification in clear cell renal cell carcinoma (ccRCC).

\section{Methods}

The Cancer Genome Atlas Kidney Renal Clear Cell Carcinoma (TCGA KIRC) dataset was downloaded and analyzed.

\section{Results}

Four prognostic hypoxia-associated IncRNAs were used for signature construction. The four IncRNAs were downregulated in high grade, advanced stage, and high-risk ccRCC. ccRCC patients in the high-risk group had a worse prognosis than those in the low-risk group. And the risk score was significantly higher in high grade and advanced stage. The signature had an independent and long-standing prognosis prediction ability up to 10-year follow-up. Notably, the risk score was significantly positively correlated with the infiltration abundances of six immune cells from the Tumor IMmune Estimation Resource (TIMER). The gene set enrichment analysis (GSEA) also suggested that the signature was involved in the metabolism and tumorigenesis, which were closely related to the hypoxic tumor microenvironment. Ultimately, a nomogram was built to predict the individual long-term survival possibility.

\section{Conclusions}

We have developed a new hypoxia-associated IncRNA signature, representing a promising tool for risk stratification tool in ccRCC. It might serve as a prognostic index to facilitate personalized counseling for treatment.

\section{Introduction}

With 208,500 new cases diagnosed worldwide each year, the renal tumor is one type of common malignancies in urinary tumors. ccRCC is the most common type of renal tumor, accounting for approximately $80 \%$ of the types of renal tumor ${ }^{1}$. The invasive features and unfavorable prognosis contribute to high morbidity and mortality of $\mathrm{ccRCC}^{2}$. With the rapid development of diagnosis and treatment, targeted therapeutic strategies have significantly improved the overall survival rate of ccRCC. However, for high-grade and advanced-stage ccRCC, the survival rate is still unsatisfactory ${ }^{3}$. Hence, effective predictive methods are urgently needed to improve the prognosis of patients with ccRCC. 
Due to the imbalance between vascular nutrient supply and the rate of tumor cell proliferation, hypoxia is an intrinsic feature of solid tumors ${ }^{4}$. Many studies have uncovered the critical roles of hypoxia in the tumor microenvironment, including cell proliferation and differentiation and tumor angiogenesis and immune infiltration. Hypoxia can activate the hypoxia-inducible factors and then induce adaptive changes within a cancer cell, which results in tumor progression and treatment resistance ${ }^{5}$. Previous studies have explored the close relations between hypoxia microenvironment and several cancers, including gastric cancer, glioblastoma, colorectal cancer, breast cancer, and hepatocellular carcinoma ${ }^{6-}$ ${ }^{10}$. However,limited evidence is reported about the possible mechanisms of hypoxia affecting the tumorigenesis, progression, and distant metastasis of ccRCC. Therefore, further efforts on the relationship between hypoxia and the biological behaviors of ccRCC are required to develop novel treatment methods.

LncRNA refers to RNA that does not encode proteins and has a length greater than 200 nucleotides. By coordinating with other molecules, IncRNA can regulate several physiological processes, and their dysfunction may contribute to pathologies, including cancer and infectious diseases ${ }^{11}$. One study by Che et al. uncovered the potential relationships between dysregulation of key IncRNA and ccRCC invasion, suggesting the critical roles played by IncRNA in the ccRCC microenvironment ${ }^{12}$.

By taking advantage of high-throughput sequencing data obtained from the TCGA database and advanced bioinformatics analyses, we carried out this present study to explore IncRNAs targeting hypoxia-related genes and further construct a hypoxia-associated prognostic signature for CcRCC patients.

\section{Results}

\section{Identification of differentially expressed hypoxia-related genes and enrichment analysis}

Sixty-one differentially expressed hypoxia-related genes were obtained (Fig. 2a) and were subjected to enrichment analysis. As listed in Fig. 2b, PID HIF1 TFPATHWAY, Metabolism of carbohydrates, response to decreased oxygen levels, blood vessel development, and extracellular structure organization were the most enriched terms, suggesting the critical roles of the hypoxia-related genes in the tumor microenvironment.

\section{IncRNAs targeting hypoxia-related genes identification and prognostic signature development}

Eighty-seven IncRNAs, targeting sixty-one hypoxia-related genes, were identified by Pearson correlation analysis and were used for subsequent prognostic signature construction. 
Under the defined criteria, 516 ccRCC samples were included and classified into a training cohort $(\mathrm{n}=$ 364) and a testing cohort $(n=152)$. We then performed univariate Cox regression analysis on the hypoxia-related IncRNAs in the training cohort and identified four IncRNAs, namely COMETT, EMX2OS, AC026462.3, and HAGLR, related to the prognosis of $\operatorname{ccRCC}(\mathrm{P}<0.01)$. Notably, the high expression of the four IncRNAs was associated with a favorable prognosis, suggesting the protective roles of these IncRNAs in patients with ccRCC.

Since the expression values of the four IncRNAs and survival data were merged, we performed the LASSO regression analysis and multivariate Cox regression analysis to develop a prognostic signature in the training cohort (Table 1). Based on the regression coefficients and gene expression, we calculated each sample's risk score by the following equation: Risk score $=-0.076 \times$ Exp. COMETT $-0.039 \times$ Exp. EMX2OS $-0.734 \times$ Exp. AC026462.3 - $0.065 \times$ Exp. HAGLR.

Table 1

The four signature IncRNAs.

\begin{tabular}{|lllllll|}
\hline LncRNA & Ensembl & Coef & HR & HR.95L & HR.95H & P-value \\
\hline COMETT & ENSG00000231210 & -0.076 & 0.927 & 0.836 & 1.028 & 0.153 \\
\hline EMX2OS & ENSG00000229847 & -0.039 & 0.962 & 0.926 & 1.000 & 0.052 \\
\hline AC026462.3 & ENSG00000260963 & -0.734 & 0.480 & 0.260 & 0.888 & 0.019 \\
\hline HAGLR & ENSG00000224189 & -0.065 & 0.937 & 0.877 & 1.002 & 0.058 \\
\hline $\begin{array}{l}\text { Coef, regression coefficient. HR, hazard ratio. 95L-95H, 95\% confidence interval of HR. P-value, } \\
\text { calculated from multivariate Cox regression analysis. }\end{array}$
\end{tabular}

Next, 364 samples in the training cohort were divided into high-risk and low-risk groups according to the median risk score of 1.102. The distribution of risk scores, the survival status, and the expression of the four IncRNAs for high- and low-risk ccRCC were shown in Fig. 3a-c. To assess the signature's prognostic value, we performed KM survival analysis and found that patients in the high-risk group had a worse prognosis than those in the low-risk group (Fig. 3d). We then produced time-dependent ROC curves to estimate the predictive power of the constructed signature. As shown in Fig. 3e, Area Under Curve (AUC) for 5 -, 7 - and 10 -year survival prediction was $0.688,0.699$, and 0.636 , respectively.

\section{Validation of prognostic hypoxia-associated IncRNAs signature}

To test the reliability and stability of the signature constructed in the training cohort, we calculated the risk score of each ccRCC in the testing and entire cohorts. We then divided the ccRCC in the two cohorts into high- and low-risk groups based on the training cohort's median risk score.

The distribution of risk scores, the survival status, and the expression of the four signature IncRNAs in the high- and low-risk samples of the testing cohort were shown in Fig. 4a-c. KM survival analysis revealed 
that patients in the high-risk group had a worse prognosis than those in the low-risk group (Fig. 4d). Timedependent ROC curves showed that the AUC for 5 -, 7 - and 10 -year survival prediction was $0.707,0.741$, and 0.837 , respectively (Fig. 4e).

The distribution of risk scores, the survival status, and the expression of the four signature IncRNAs in the high- and low-risk samples of the entire cohort were shown in Fig. 5a-c. KM survival analysis revealed that patients in the high-risk group had a worse prognosis than those in the low-risk group (Fig. 5d). Timedependent ROC curves showed that the AUC for 5-, 7- and 10-year survival prediction was $0.696,0.716$, and 0.7 (Fig. 5e).

To explore the performance of the constructed signature in progression-free survival, we performed KM analysis and generated time-dependent ROC curves. Results showed that patients with CCRCC in the highrisk group had worse PFS than those in the low-risk group. The AUC for 5-, 7- and 10-year survival prediction was $0.711,0.703$, and 0.665 (see Supplementary Fig. S1 online).

Generally, the four hypoxia-associated IncRNA signature presented significantly prognostic value and good stability in ccRCC.

\section{Independent analysis of the signature and clinicopathological characteristics}

Univariate Cox regression analysis and multivariate Cox regression analysis were performed on the four hypoxia-associated IncRNA signature and clinicopathological characteristics (age, gender, grade, and stage) to determine whether the prediction ability of the signature was independent of clinicopathological factors. As shown in Table 2, the signature showed independent prognostic prediction ability in three cohorts simultaneously. Besides, grade and stage were found to be two independent clinicopathological predictors of ccRCC. 
Table 2

Independent prognostic analysis in three cohorts of TCGA KIRC dataset.

\begin{tabular}{|c|c|c|c|c|c|c|c|c|c|}
\hline \multirow[t]{2}{*}{ Cohort } & \multirow[t]{2}{*}{ Parameters } & \multicolumn{4}{|c|}{ uniCox } & \multicolumn{4}{|c|}{ multiCox } \\
\hline & & $\mathrm{HR}$ & HR.95L & HR.95H & $\begin{array}{l}\mathrm{P}- \\
\text { value }\end{array}$ & $\mathrm{HR}$ & HR.95L & HR.95H & $\begin{array}{l}\text { P- } \\
\text { value }\end{array}$ \\
\hline \multirow[t]{5}{*}{ train } & age & 1.026 & 1.011 & 1.041 & $\begin{array}{l}< \\
0.001\end{array}$ & 1.030 & 1.013 & 1.048 & $\begin{array}{l}<.001 \\
0.001\end{array}$ \\
\hline & gender & 0.884 & 0.610 & 1.283 & 0.517 & 0.889 & 0.601 & 1.314 & 0.556 \\
\hline & grade & 2.092 & 1.648 & 2.656 & $\begin{array}{l}<.001 \\
0\end{array}$ & 1.321 & 1.007 & 1.732 & 0.044 \\
\hline & stage & 1.866 & 1.590 & 2.191 & $\begin{array}{l}< \\
0.001\end{array}$ & 1.648 & 1.372 & 1.979 & $\begin{array}{l}< \\
0.001\end{array}$ \\
\hline & riskScore & 2.274 & 1.729 & 2.992 & $\begin{array}{l}< \\
0.001\end{array}$ & 1.606 & 1.191 & 2.165 & 0.002 \\
\hline \multirow[t]{5}{*}{ test } & age & 1.031 & 1.005 & 1.058 & 0.019 & 1.029 & 1.000 & 1.059 & 0.051 \\
\hline & gender & 1.171 & 0.647 & 2.118 & 0.602 & 1.235 & 0.678 & 2.251 & 0.490 \\
\hline & grade & 2.731 & 1.821 & 4.095 & $\begin{array}{l}< \\
0.001\end{array}$ & 1.527 & 0.942 & 2.476 & 0.086 \\
\hline & stage & 1.876 & 1.480 & 2.377 & $\begin{array}{l}< \\
0.001\end{array}$ & 1.521 & 1.144 & 2.022 & 0.004 \\
\hline & riskScore & 2.388 & 1.601 & 3.562 & $\begin{array}{l}< \\
0.001\end{array}$ & 1.655 & 1.083 & 2.531 & 0.020 \\
\hline \multirow[t]{5}{*}{ entire } & age & 1.027 & 1.014 & 1.040 & $\begin{array}{l}< \\
0.001\end{array}$ & 1.031 & 1.016 & 1.045 & $\begin{array}{l}< \\
0.001\end{array}$ \\
\hline & gender & 0.961 & 0.702 & 1.316 & 0.806 & 0.981 & 0.710 & 1.354 & 0.907 \\
\hline & grade & 2.254 & 1.836 & 2.767 & $\begin{array}{l}< \\
0.001\end{array}$ & 1.361 & 1.077 & 1.720 & 0.010 \\
\hline & stage & 1.876 & 1.643 & 2.142 & $\begin{array}{l}< \\
0.001\end{array}$ & 1.613 & 1.385 & 1.878 & $\begin{array}{l}< \\
0.001\end{array}$ \\
\hline & riskScore & 2.309 & 1.843 & 2.893 & $\begin{array}{l}< \\
0.001\end{array}$ & 1.645 & 1.293 & 2.094 & $\begin{array}{l}< \\
0.001\end{array}$ \\
\hline
\end{tabular}

Next, we analyzed the relationship between the four hypoxia-associated IncRNA signature and clinicopathological characteristics. The results showed that patients with high grade (III-IV) and advanced stage (III-IV) had higher risk scores (Fig. 6a). Stratified analysis was further carried out between the four hypoxia-associated IncRNA signature and the two clinicopathological independent predictors (grade and stage). The results of KM curves and time-dependent ROC curves showed that the four hypoxia- 
associated IncRNA signature had significant prognostic value and reliability for CcRCC with the same grade and stage (Fig. $6 \mathrm{~b}-\mathrm{C}$ ). These results suggested the possible relationship between the signature and progression of ccRCC.

We also analyzed the relationships between expression values of the four signature IncRNAs and grade and stage. As shown in Fig. 7, all the four IncRNAs were associated with high grade (except for COMETT) and advanced stage, indicating the prognostic values of the four hypoxia-associated IncRNAs.

\section{Correlation analysis between risk score and infiltration levels of immune cells}

Next, we performed a correlation analysis between the risk scores of the signature and infiltration abundances of the six immune cells (B cells, CD4 T cells, CD8 T cells, Dendritic cells, Macrophages, and Neutrophils) obtained from TIMER. The results revealed that all the immune cells showed a significantly positive correlation with the risk score (Fig. 8a). Using the ESTIMATE algorithm, we obtained the stromal and immune scores of each ccRCC sample and found that the high-risk group had higher stromal and immune scores than the low-risk group (Fig. 8b). These findings further prove the correlation between hypoxia microenvironment and tumor immune activity, providing evidences for novel tumor therapeutic strategies.

GSEA was used to conduct key gene ontology term and pathway enrichment analysis of high and lowrisk score ccRCC samples. Genes associated with high-risk score were mainly enriched in metabolism, carcinogenesis, complement, and coagulation cascades (Fig. 8c).

\section{Construction of a nomogram based on the four hypoxia- associated IncRNA signature}

Based on the prognostic signature and clinicopathological factors, we constructed a nomogram to predict the 5-,7-, and 10-year overall survival of cCRCC (Fig. 9a). Time-dependent ROC curves showed the nomogram's good discrimination with an AUC of 0.604, 0.608, and 0.769 at 5-, 7-, and 10-year follow-up (Fig. 9b). Additionally, we generated the calibration plots and performed the decision curve analysis to demonstrate the good predictive effect and clinical utility of the nomogram (Fig. 9c-d). Ultimately, a multiparameter ROC curve was generated to show the superior discrimination of the nomogram at 10-year follow-up (Fig. 9e).

\section{Discussion}

In this study, we developed a hypoxia-associated four-IncRNA signature closely relating to long-term prognosis of patients with ccRCC, based on the IncRNAs targeting differentially expressed hypoxiarelated genes. The signature was further validated in the testing cohort and the entire cohort from the TCGA KIRC dataset. KM survival analysis in the three cohorts showed that patients in the high-risk group had poor prognosis while those in the low-risk group had favorable prognosis, indicating the good 
prognostic ability of the signature. Additionally, AUC for 5-, 7-, and 10-year follow-up in the three cohorts suggested stable performance in distinguishing different risk-level ccRCC. Notably, we proposed a risk score calculating formula by which we can predict the risk of patients with ccRCC once the expression values of the four IncRNAs were obtained. In correlation analysis between signature and clinicopathological factors, we found that risk score was significantly higher in advanced stage (III-IV) and high grade (III-IV). Stratified analysis in the same stage and grade (I-II or III-IV) also demonstrated the good performance of the constructed signature. In general, the four-IncRNA signature demonstrated pretty good prognostic and discriminable ability for ccRCC.

Subsequently, the correlation between risk score and immune infiltration showed that higher risk score was associated with high immune infiltration levels in the microenvironment of CCRCC, which was consistent with previous studies ${ }^{13}$. GSEA enrichment analysis showed gene set in the high-risk group was mainly enriched in metabolism and tumorigenesis, suggesting the possible mechanism of hypoxia affecting the tumor microenvironment ${ }^{14}$.

Based on this signature and other clinicopathological factors, we further constructed a nomogram to predict the up to 10-year survival probability of patients with ccRCC. More importantly, decision curve analysis further proves the superior clinical utility of the constructed nomogram to grade and stage classification system.

The four signature IncRNAs, including COMETT, EMX2OS, AC026462.3, and HAGLR, were differentially expressed between cCRCC and normal samples. Downregulation of these four IncRNAs was associated with advanced stage, high grade and poor prognosis, suggesting the protective roles in tumorigenesis, progression, and metastasis. COMETT, also known as $L I N C 01510$, has diagnostic and prognostic values in ccRCC, as reported previously ${ }^{15,16}$. Ma et al. also reported that low expression of COMETT in ccRCC was associated with poor prognosis, which was consistent with our study results. Furthermore, they found that COMETT acted as a tumor suppressor in ccRCC tumorigenesis by inhibiting Wnt/ $\beta$-catenin signaling ${ }^{17}$. The biological behaviors of COMETT were also studied in papillary thyroid carcinoma, colorectal cancer, and non-small cell lung cancer ${ }^{18-20}$. EMX2OS, an enhancer RNA, was upregulated in low-risk ccRCC and significantly associated with low grade, early stage, and favorable prognosis ${ }^{21}$. Protective roles of this IncRNA were also found and validated in classical papillary thyroid cancer ${ }^{22}$, prostate cancer ${ }^{23}$. The role of EMX2OS in hypoxia was also reported in myalgic encephalomyelitis/chronic fatigue syndrome by Yang et al. ${ }^{24}$. High expression of HAGLR suggested a favorable prognosis for $\mathrm{CCRCC}$. While the expression of HAGLR was upregulated in hepatocellular carcinoma and associated with tumor proliferative and metastatic ${ }^{25}$. Similar findings in colon cancer and esophageal cancer were reported by Sun and Yang et al. ${ }^{26,27}$. Interestingly, HAGLR coordinating with miRNA-19a-3p/TGFBR2 could promote the healing process of a femoral neck fracture. Additionally, HAGLR showed strong diagnostic power for atrial fibrillation, acting as a competing endogenous RNA ${ }^{28}$. 
The main limitation of the current study lacks validation in an independent dataset or clinical ccRCC samples. Our results should be further validated by prospective studies in multiple centers.

In conclusion, We have generated a four-IncRNA prognostic risk score model consisting of COMETT, EMX2OS, AC026462.3, and HAGLR. The predictive nomograms integrating both the four-IncRNA signature and the conventional clinical-pathological risk factors were robust in predicting 5-, 7- and 10year survival probabilities.

\section{Methods}

Figure 1 presents the workflow of our study.

\section{Data acquisition}

The gene expression data of level 3 RNA-seq fragments per kilobase million (FPKM) dataset and clinical data were retrieved from TCGA KIRC (https://portal.gdc.cancer.gov/) and UCSC Xena (https://xenabrowser.net/), respectively.

Two hundred hypoxia-related genes were downloaded from the hallmark gene sets of the Molecular Signatures Database (M5891, https://www.gsea-msigdb.org/gsea/msigdb/).

\section{Identification of differentially expressed hypoxia-related genes and enrichment analysis}

The expression profiles of the hypoxia-related genes were extracted from the TCGA KIRC dataset. Then, we applied the limma R package to select the differentially expressed hypoxia-related genes between ccRCC samples and normal renal samples ${ }^{29}$. Adjusted P-value (adj. P-value) of 0.05 and $|\log F C| \geq 1$ were set as the cut-off criteria. The online tool Metascape was used for enrichment analysis (https://metascape.org/gp/index.html) ${ }^{30}$.

\section{Identification of IncRNAs targeting hypoxia-related genes}

To screen out the IncRNAs targeting differentially expressed hypoxia-related genes, we used Pearson correlation analysis between the expression profiles of all IncRNAs, and the differentially expressed hypoxia-related genes. $|\mathrm{R}|>0.6$ and P-value $<0.01$ were set as the cutoff criteria.

\section{Construction of a hypoxia-associated IncRNAs signature}

The inclusion criteria for ccRCC samples in IncRNA signature construction and validation included: (1) samples with complete IncRNA expression levels, and (2) samples with total survival time over 30 days. Subsequently, the eligible ccRCC samples were randomly divided into the training and testing cohorts on the ratio of 7:3 by caret $R$ package. 
First, we performed univariate Cox regression analysis in the training cohort to screen the prognostic hypoxia-associated IncRNAs by the cutoff value $(P<0.01)$. Then, we performed the Least Absolute Shrinkage and Selector Operation (LASSO) analysis, which can reduce the estimation variance and provide an explicable final model. Then, we used the multivariate Cox regression analysis to establish a prognostic signature. Furthermore, we calculated the risk score of each ccRCC using the formula: Risk score $=$ ExplncRNA1 $\times$ CoeflncRNA1 + ExplncRNA2 $\times$ CoeflncRNA2 $+\ldots$ ExplncRNA $(n) \times$ CoeflncRNA $\square n \rrbracket$. In this equation, "ExplncRNA" represented the expression level of a IncRNA, and "CoeflncRNA" was the regression coefficient of a IncRNA calculated by the multivariate Cox regression analysis. Next, the median value of the risk score was set as the threshold to divide the training cohort into high- and low-risk groups. The Kaplan-Meier (KM) survival curve by log-rank test and time-dependent receiver operating characteristic (ROC) curve were plotted to assess the signature's predictive ability. Further, the signature's stability and reliability were verified in the testing, and the entire cohorts using similar methods. The glmnet, survival, and survminer R packages were used for these analyses.

We then used the univariate and multivariate Cox regression analyses to assess the signature's independent predictive ability based on the signature and clinicopathological characteristics in the three subgroups of the TCGA KIRC dataset. We also performed the stratified analysis to analyze the clinicopathological factors with significance $(P<0.05)$.

\section{Assessment of immune cell infiltration and immune microenvironment}

Published studies have proposed the close relationships between hypoxia, tumor immunity, and immune microenvironment. Hence, we performed a correlation analysis between risk score and infiltration abundances of six immune cells downloaded from the TIMER (https://cistrome.shinyapps.io/timer/) ${ }^{31}$. Additionally, the ESTIMATE algorithm was used to assess the immune infiltration in ccRCC ${ }^{32}$.

By clusterProfiler R package, GSEA was performed on the expression matrices of high- and low-risk groups to uncover the possible mechanism of risk level affecting hypoxia and tumor microenvironment 33.

\section{Development and evaluation of a nomogram}

A nomogram of signature and other independent clinicopathological characteristics was built by the rms $\mathrm{R}$ package. Then, time-dependent ROC curves and calibration plots were produced to validate the nomogram's discrimination and predictive accuracy, respectively.

\section{Abbreviations}

IncRNA, long non-coding; ccRCC, clear cell renal cell carcinoma; TIMER, Tumor IMmune Estimation Resource; TCGA KIRC, The Cancer Genome Atlas Kidney Renal Clear Cell Carcinoma; adj. P-value, Adjusted P-value; FPKM, fragments per kilobase million; logFC, log (fold change); LASSO, Least Absolute 
Shrinkage and Selector Operation; ROC, receiver operating characteristic; GSEA, gene set enrichment analysis; AUC, Area Under Curve. ESTIMATE, Estimation of STromal and Immune cells in MAlignant Tumours using Expression data; COMETT, Cytosolic Oncogenic Antisense To MET Transcript; EMX2OS, EMX2 Opposite Strand/Antisense RNA; HAGLR, HOXD Antisense Growth-Associated Long Non-Coding RNA; KM, Kaplan-Meier; TGFBR2, Transforming Growth Factor Beta Receptor 2.

\section{Declarations}

\section{Funding}

This work was supported by Chongqing Science and Technology Commission (cstc2015shmszx120067).

\section{Data availability}

The datasets analysed during the current study are available in The Cancer Genome Atlas (https://portal.gdc.cancer.gov/).

\section{Acknowledgements}

We appreciate the works by the Cancer Genome Atlas database (TCGA) dataset for the source of data used in our study.

\section{Authors contributions}

CHL and CG: conceptualization. CHL, PY and JXX: data curation and methodology. PY and JXX: data analysis and visualization. CHL: writing review \& editing. All authors contributed to manuscript revision, read and approved the submitted version.

\section{Competing interests}

The authors declare no competing interests..

\section{References}

1 Attalla, K., Weng, S., Voss, M. H. \& Hakimi, A. A. Epidemiology, Risk Assessment, and Biomarkers for Patients with Advanced Renal Cell Carcinoma. Urol Clin North Am47, 293-303, doi:10.1016/j.ucl.2020.04.002 (2020).

2 Ren, X. Et al. Upregulation of KIF20A promotes tumor proliferation and invasion in renal clear cell carcinoma and is associated with adverse clinical outcome. Aging (Albany NY)12, 
doi:10.18632/aging.202153 (2020).

3 Yuan, Y. Et al. Expression and prognostic significance of fatty acid synthase in clear cell renal cell carcinoma. Pathol Res Pract216, 153227, doi:10.1016/j.prp.2020.153227 (2020).

4 Muz, B., de la Puente, P., Azab, F. \& Azab, A. K. The role of hypoxia in cancer progression, angiogenesis, metastasis, and resistance to therapy. Hypoxia (Auckl)3, 83-92, doi:10.2147/HP.S93413 (2015).

5 Eales, K. L., Hollinshead, K. E. R. \& Tennant, D. A. Hypoxia and metabolic adaptation of cancer cells. Oncogenesis5, e190-e190, doi:10.1038/oncsis.2015.50 (2016).

6 Colwell, N. Et al. Hypoxia in the glioblastoma microenvironment: shaping the phenotype of cancer stemlike cells. Neuro Onco/19, 887-896, doi:10.1093/neuonc/now258 (2017).

7 Guo, Y. Et al. Hypoxia-inducible factors in hepatocellular carcinoma (Review). Oncol Rep43, 3-15, doi:10.3892/or.2019.7397 (2020).

8 Liu, L. Et al. Hypoxia Promotes Gastric Cancer Malignancy Partly through the HIF-1a Dependent Transcriptional Activation of the Long Non-coding RNA GAPLINC. Front Physio/7, 420-420, doi:10.3389/fphys.2016.00420 (2016).

$9 \mathrm{Mi}$, Y. Et al. Hypoxic colorectal cancer cells promote metastasis of normoxic cancer cells depending on IL-8/p65 signaling pathway. Cell Death \& Disease11, 610, doi:10.1038/s41419-020-02797-z (2020).

10 Rausch, L. K., Netzer, N. C., Hoegel, J. \& Pramsohler, S. The Linkage between Breast Cancer, Hypoxia, and Adipose Tissue. Front Oncol7, 211-211, doi:10.3389/fonc.2017.00211 (2017).

11 Zhang, L., Xu, X. \& Su, X. Noncoding rnas in cancer immunity: functions, regulatory mechanisms, and clinical application. Molecular Cancer19, 48, doi:10.1186/s12943-020-01154-0 (2020).

12 Che, J., Yao, X., Wang, C., Zheng, J. \& Guo, C. Hypoxia promoted renal cell carcinoma cell migration through regulating Incrna-ENST00000574654. 1. Am J Transl Res12, 3884 (2020).

13 Hu, D., Zhou, M. \& Zhu, X. Deciphering Immune-Associated Genes to Predict Survival in Clear Cell Renal Cell Cancer. Biomed Res Int2019, 2506843-2506843, doi:10.1155/2019/2506843 (2019).

14 Wheaton, W. W. \& Chandel, N. S. Hypoxia. 2. Hypoxia regulates cellular metabolism. Am J Physiol Cell Physio/300, C385-C393, doi:10.1152/ajpcell.00485.2010 (2011).

15 Cheng, G. Et al. A cluster of long non-coding rnas exhibit diagnostic and prognostic values in renal cell carcinoma. Aging (Albany NY)11, 9597-9615, doi:10.18632/aging.102407 (2019).

16 Zeng, J. H. Et al. Prognosis of clear cell renal cell carcinoma (ccrcc) based on a six-Incrna-based risk score: an investigation based on RNA-sequencing data. J Trans/ Med17, 281, doi:10.1186/s12967-0192032-y (2019). 
$17 \mathrm{Ma}, \mathrm{B}$. Et al. LINC01510 suppresses cell proliferation and invasion by inhibiting Wnt/ $\beta$-catenin signaling in renal cell carcinoma. Biochem Biophys Res Commun505, 7-12, doi:10.1016/j.bbrc.2018.08.167 (2018).

$18 \mathrm{Li}$, J. \& Wei, L. Increased expression of LINC01510 predicts poor prognosis and promotes malignant progression in human non-small cell lung cancer. Biomed Pharmacother109, 519-529, doi:10.1016/j.biopha.2018.10.136 (2019).

19 Li, Q., Wang, X. J. \& Jin, J. H. SOX2-induced upregulation of Incrna LINC01510 promotes papillary thyroid carcinoma progression by modulating mir-335/SHH and activating Hedgehog pathway. Biochem Biophys Res Commun520, 277-283, doi:10.1016/j.bbrc.2019.09.138 (2019).

20 Zhou, J. F. Et al. Long noncoding RNA LINC01510 is highly expressed in colorectal cancer and predicts favorable prognosis. Eur Rev Med Pharmacol Sci22, 7710-7715, doi:10.26355/eurrev_201811_16392 (2018).

21 Jiang, H., Chen, H., Wan, P., Song, S. \& Chen, N. Downregulation of enhancer RNA EMX2OS is associated with poor prognosis in kidney renal clear cell carcinoma. Aging (Albany NY)12, doi:10.18632/aging.202151 (2020).

22 Gu, Y., Feng, C., Liu, T., Zhang, B. \& Yang, L. The downregulation of Incrna EMX2OS might independently predict shorter recurrence-free survival of classical papillary thyroid cancer. Plos One13, e0209338, doi:10.1371/journal.pone.0209338 (2018).

23 Wang, Z. Et al. Lncrna EMX20S, Regulated by TCF12, Interacts with FUS to Regulate the Proliferation, Migration and Invasion of Prostate Cancer Cells Through the cgmp-PKG Signaling Pathway. Onco Targets Ther13, 7045-7056, doi:10.2147/ott.S243552 (2020).

24 Yang, C. A. Et al. The expression signature of very long non-coding RNA in myalgic encephalomyelitis/chronic fatigue syndrome. J Trans/ Med16, 231, doi:10.1186/s12967-018-1600-x (2018).

25 Li, Y. H., Huang, G. M., Wang, W. \& Zang, H. L. Lncrna HAGLR exacerbates hepatocellular carcinoma through negatively regulating mir-6785-5p. Eur Rev Med Pharmacol Sci24, 9353-9360, doi:10.26355/eurrev_202009_23018 (2020).

26 Sun, W. Et al. Lnc HAGLR Promotes Colon Cancer Progression Through Sponging mir-185-5p and Activating CDK4 and CDK6 in vitro and in vivo. Onco Targets Ther13, 5913-5925, doi:10.2147/ott.S246092 (2020).

27 Yang, C. Et al. Long noncoding RNA HAGLR acts as a microrna-143-5p sponge to regulate epithelialmesenchymal transition and metastatic potential in esophageal cancer by regulating LAMP3. Faseb j33, 10490-10504, doi:10.1096/fj.201802543RR (2019). 
28 Qian, C., Li, H., Chang, D., Wei, B. \& Wang, Y. Identification of functional Incrnas in atrial fibrillation by integrative analysis of the Incrna-mrna network based on competing endogenous rnas hypothesis. $J$ Cell Physio/234, 11620-11630, doi:10.1002/jcp.27819 (2019).

29 Ritchie, M. E. Et al. Limma powers differential expression analyses for RNA-sequencing and microarray studies. Nucleic acids research43, e47-e47 (2015).

30 Zhou, Y. Et al. Metascape provides a biologist-oriented resource for the analysis of systems-level datasets. Nat Commun10, 1523, doi:10.1038/s41467-019-09234-6 (2019).

$31 \mathrm{Li}, \mathrm{T}$. Et al. TIMER: a web server for comprehensive analysis of tumor-infiltrating immune cells. Cancer research77, e108-e110 (2017).

32 Yoshihara, K. Et al. Inferring tumour purity and stromal and immune cell admixture from expression data. Nature communications4, 1-11 (2013).

33 Yu, G., Wang, L.-G., Han, Y. \& He, Q.-Y. Clusterprofiler: an R package for comparing biological themes among gene clusters. Omics: a journal of integrative biology16, 284-287 (2012).

\section{Figures}

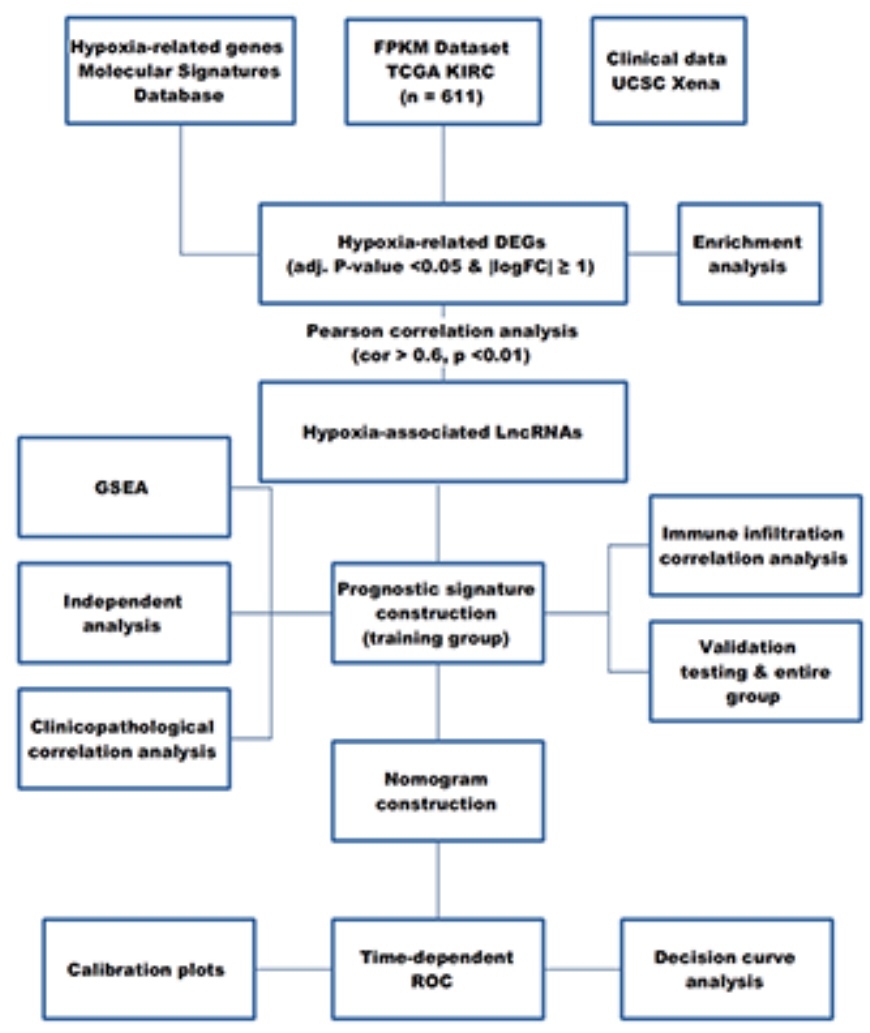

\section{Figure 1}


The workflow of this study. FPKM, Fragments Per Kilobase Million. TCGA KIRC, The Cancer Genome Atlas Kidney Renal Clear Cell Carcinoma. DEG, differentially expressed gene. adj. P-value, adjusted P-value. logFC, log (fold change). IncRNA, long non-coding RNA. GSEA, Gene Set Enrichment Analysis. ROC, receiver operating characteristic curve.

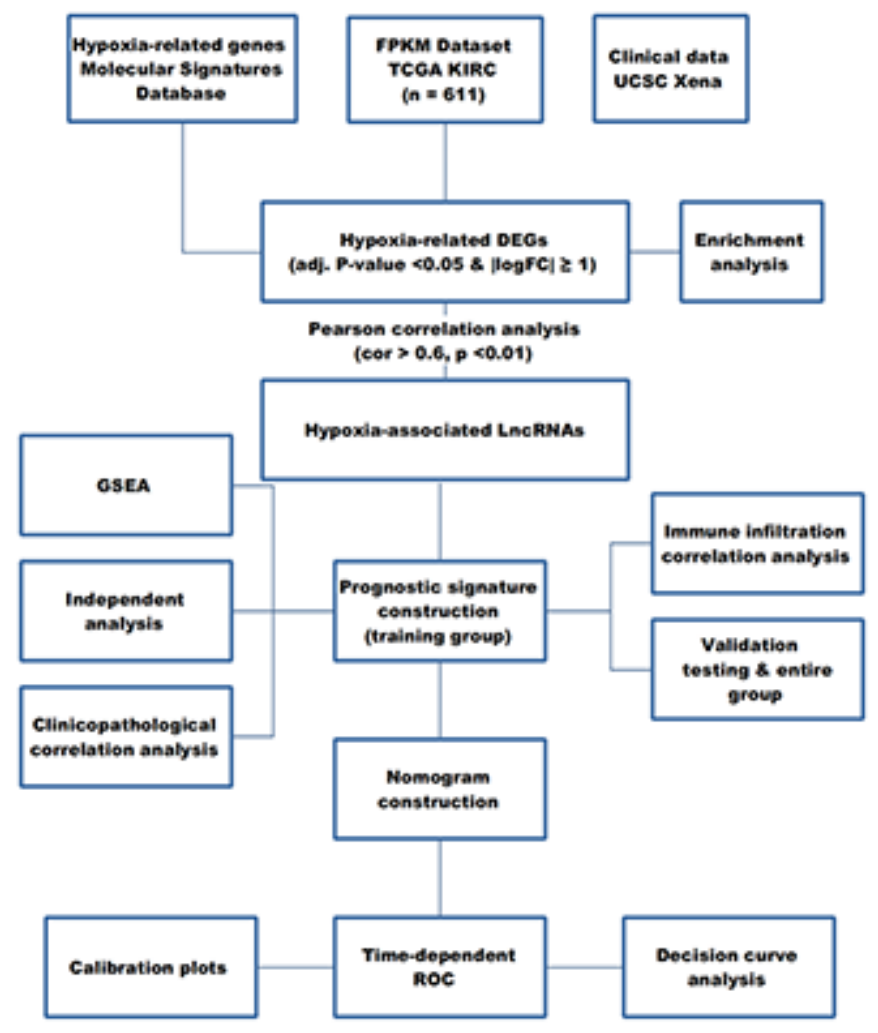

\section{Figure 1}

The workflow of this study. FPKM, Fragments Per Kilobase Million. TCGA KIRC, The Cancer Genome Atlas Kidney Renal Clear Cell Carcinoma. DEG, differentially expressed gene. adj. P-value, adjusted P-value. $\log F C$, log (fold change). IncRNA, long non-coding RNA. GSEA, Gene Set Enrichment Analysis. ROC, receiver operating characteristic curve. 

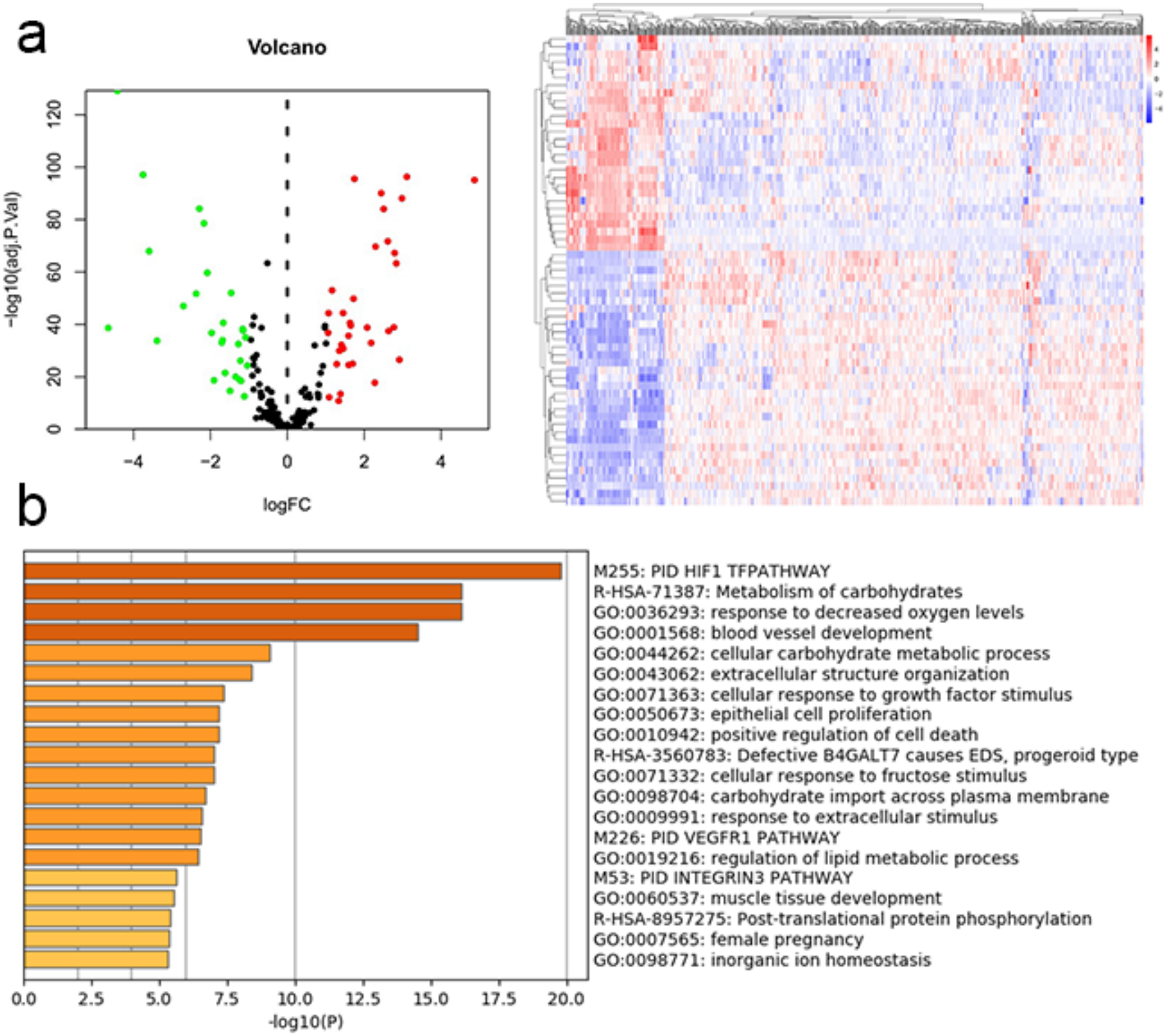

M255: PID HIF 1 TFPATHWAY

R-HSA-71387: Metabolism of carbohydrates

GO:0036293: response to decreased oxygen levels

GO:0001568: blood vessel development

GO:0044262: cellular carbohydrate metabolic process

GO:0043062: extracellular structure organization

GO:0071363: cellular response to growth factor stimulus

GO:0050673: epithelial cell proliferation

GO:0010942: positive regulation of cell death

R-HSA-3560783: Defective B4GALT7 causes EDS, progeroid type

GO:0071332: cellular response to fructose stimulus

GO:0098704: carbohydrate import across plasma membrane

GO:0009991: response to extracellular stimulus

M226: PID VEGFR1 PATHWAY

GO:0019216: regulation of lipid metabolic process

M53: PID INTEGRIN3 PATHWAY

GO:0060537: muscle tissue development

R-HSA-8957275: Post-translational protein phosphorylation

GO:0007565: female pregnancy

GO:0098771: inorganic ion homeostasis

\section{Figure 2}

DE-hypoxia-related genes identification and enrichment analysis. a Volcano plot and heatmap of the DEhypoxia-related genes identified from TCGA KIRC dataset. In the volcano plot, red dots represent upregulated genes, and green dots represent downregulated ones. b Enrichment analysis of DE-hypoxiarelated genes by online tool Metascape (https://metascape.org/). The top 20 enriched terms are listed, and the color of the bar represents the value of -log10(P-value). 

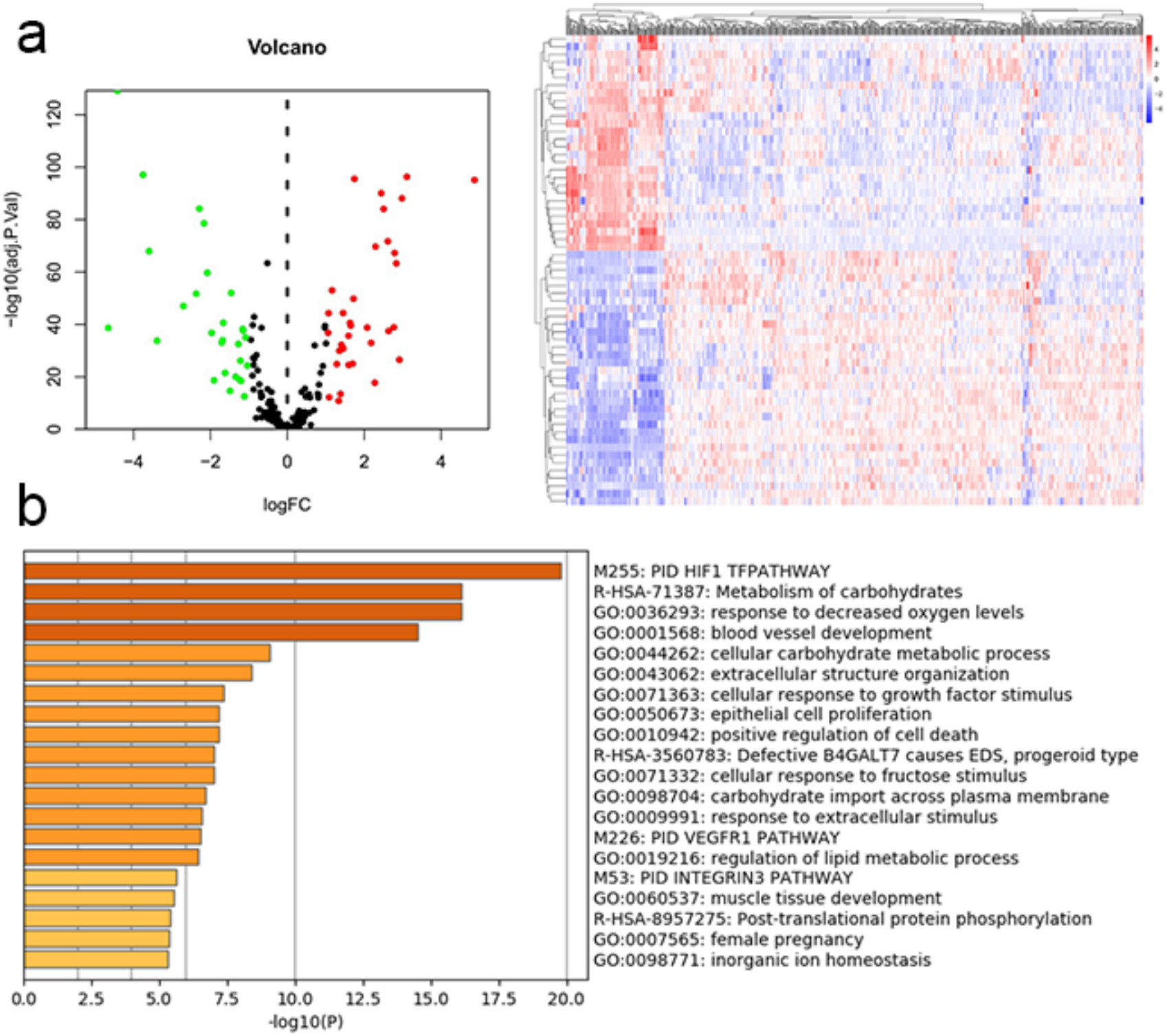

M255: PID HIF 1 TFPATHWAY

R-HSA-71387: Metabolism of carbohydrates

GO:0036293: response to decreased oxygen levels

GO:0001568: blood vessel development

GO:0044262: cellular carbohydrate metabolic process

GO:0043062: extracellular structure organization

GO:0071363: cellular response to growth factor stimulus

GO:0050673: epithelial cell proliferation

GO:0010942: positive regulation of cell death

R-HSA-3560783: Defective B4GALT7 causes EDS, progeroid type

GO:0071332: cellular response to fructose stimulus

GO:0098704: carbohydrate import across plasma membrane

GO:0009991: response to extracellular stimulus

M226: PID VEGFR1 PATHWAY

GO:0019216: regulation of lipid metabolic process

M53: PID INTEGRIN3 PATHWAY

GO:0060537: muscle tissue development

R-HSA-8957275: Post-translational protein phosphorylation

GO:0007565: female pregnancy

GO:0098771: inorganic ion homeostasis

\section{Figure 2}

DE-hypoxia-related genes identification and enrichment analysis. a Volcano plot and heatmap of the DEhypoxia-related genes identified from TCGA KIRC dataset. In the volcano plot, red dots represent upregulated genes, and green dots represent downregulated ones. b Enrichment analysis of DE-hypoxiarelated genes by online tool Metascape (https://metascape.org/). The top 20 enriched terms are listed, and the color of the bar represents the value of -log10(P-value). 

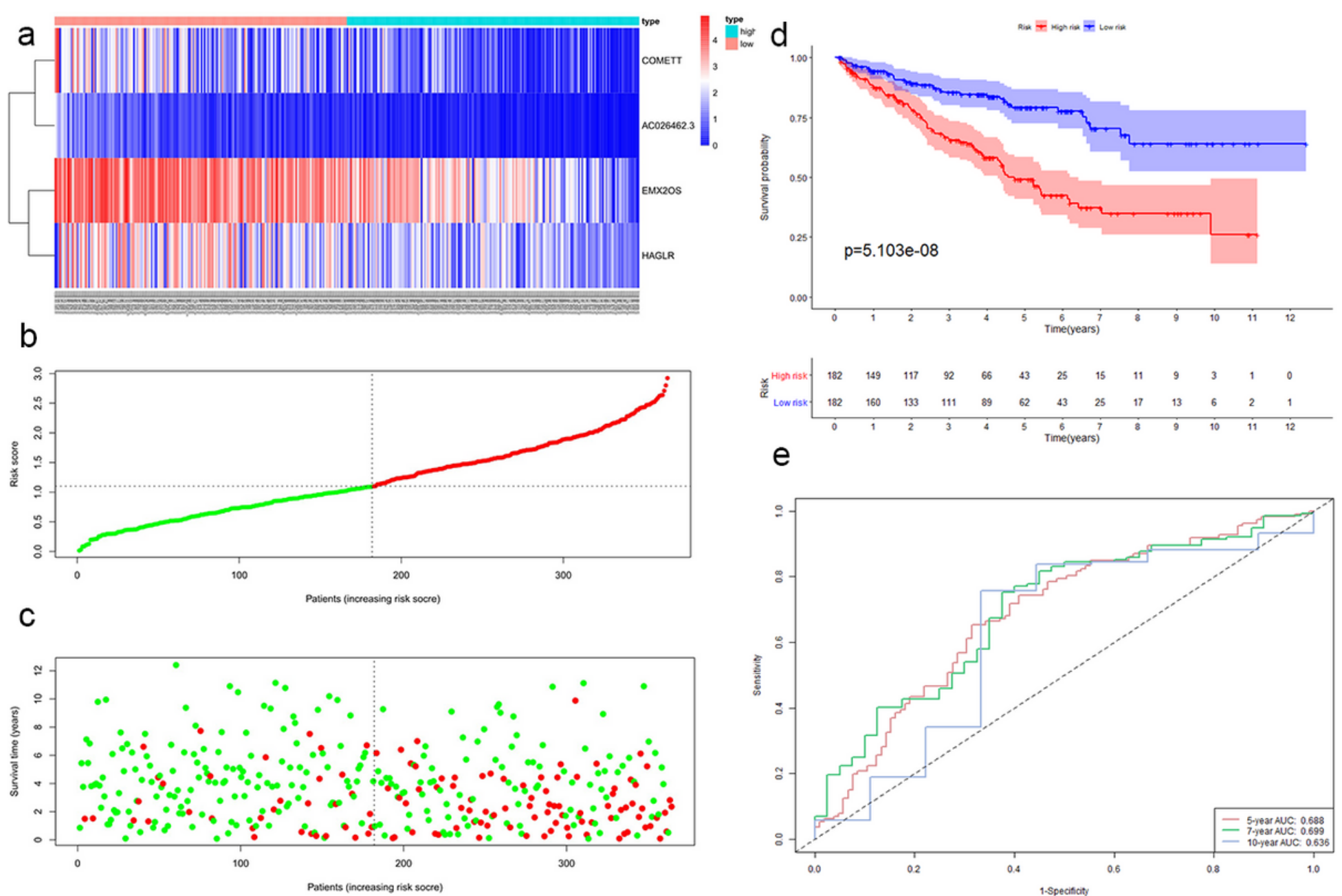

\section{Figure 3}

Four hypoxia-related IncRNA signature validated in the training cohort $(\mathrm{n}=364)$. a Expression values of the four signature IncRNAs. The color from red to blue corresponds to the expression of a IncRNA from high to low. The risk score (b) and survival status (c) of each ccRCC patient distributed in low- and highrisk group. $d \mathrm{KM}$ survival analysis between the high-risk group and low-risk group. The lower layer demonstrates the number of alive ccRCC patients in each group. e Time-dependent ROC curves of the signature. 

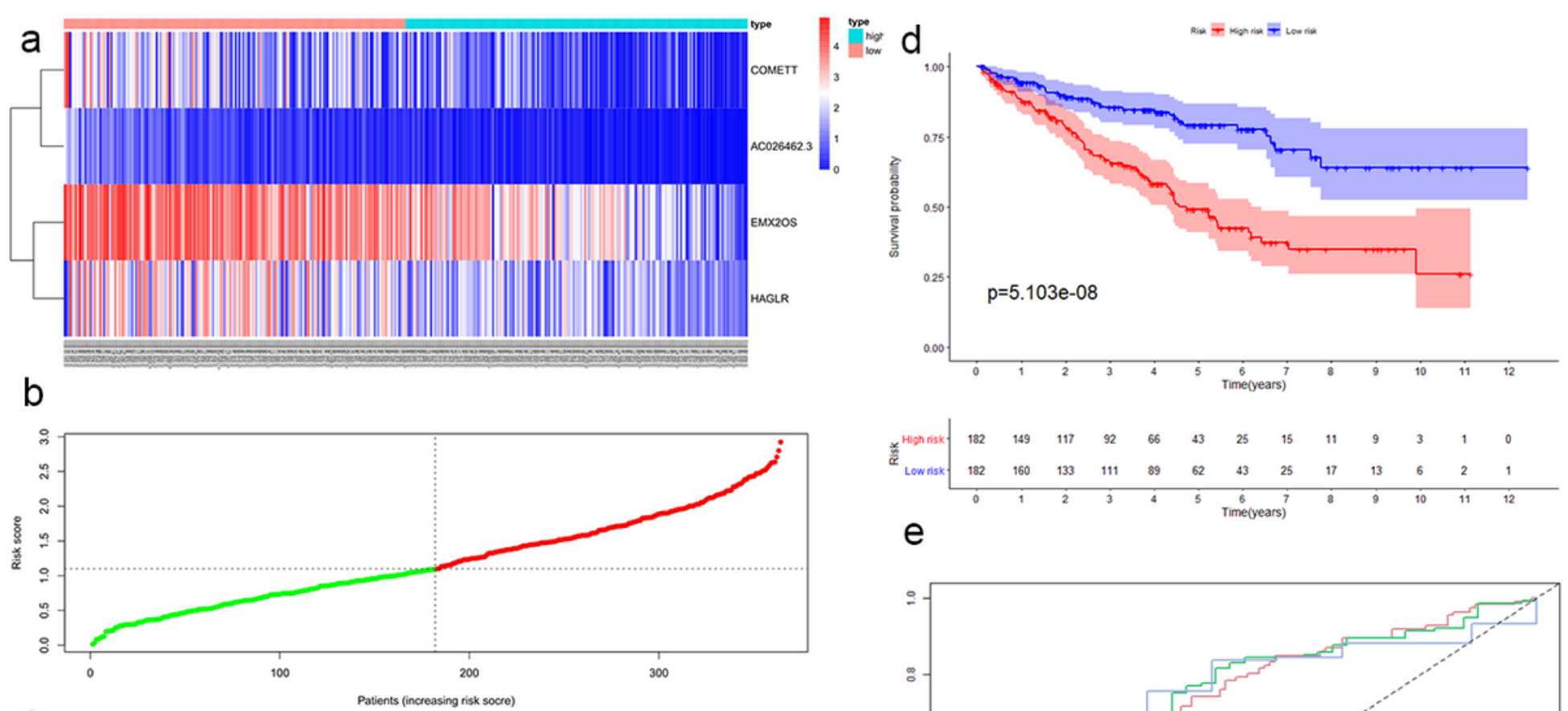

e

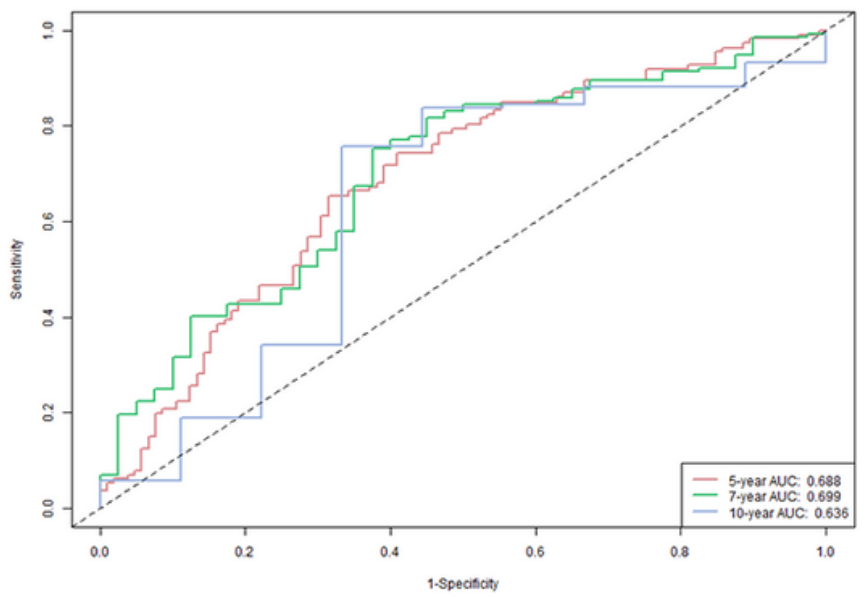

Figure 3

Four hypoxia-related IncRNA signature validated in the training cohort $(\mathrm{n}=364)$. a Expression values of the four signature IncRNAs. The color from red to blue corresponds to the expression of a IncRNA from high to low. The risk score (b) and survival status (c) of each ccRCC patient distributed in low- and highrisk group. d KM survival analysis between the high-risk group and low-risk group. The lower layer demonstrates the number of alive ccRCC patients in each group. e Time-dependent ROC curves of the signature. 

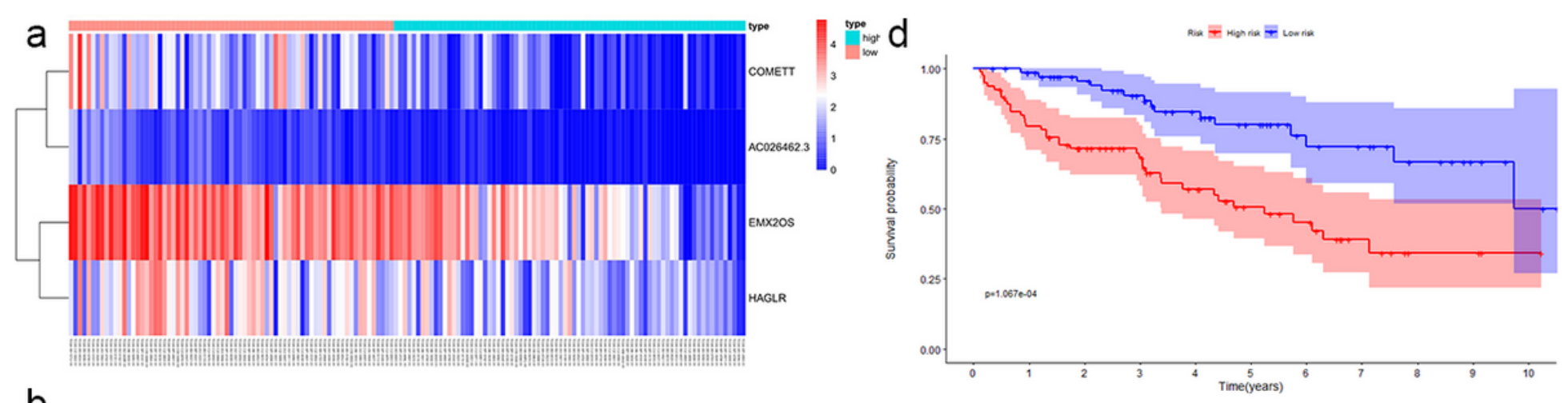

b
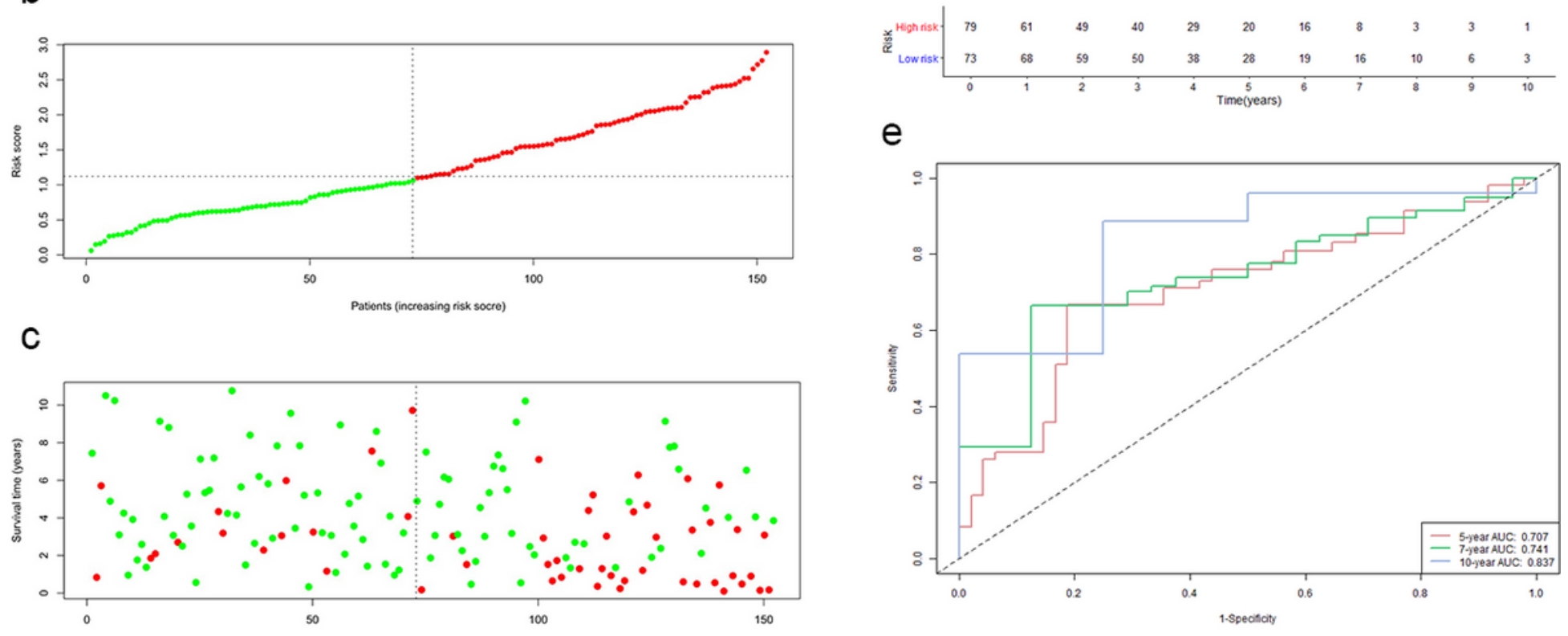

C

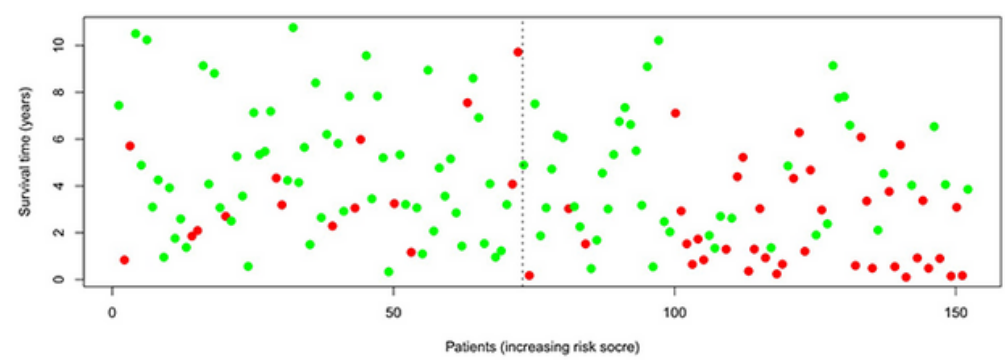

Figure 4

Four hypoxia-related IncRNA signature validated in the testing cohort $(n=152)$. a Expression values of the four signature IncRNAs. The color from red to blue corresponds to the expression of a IncRNA from high to low. The risk score (b) and survival status (c) of each ccRCC patient distributed in low- and highrisk group. d KM survival analysis between the high-risk group and low-risk group. The lower layer demonstrates the number of alive ccRCC patients in each group. e Time-dependent ROC curves of the signature. 

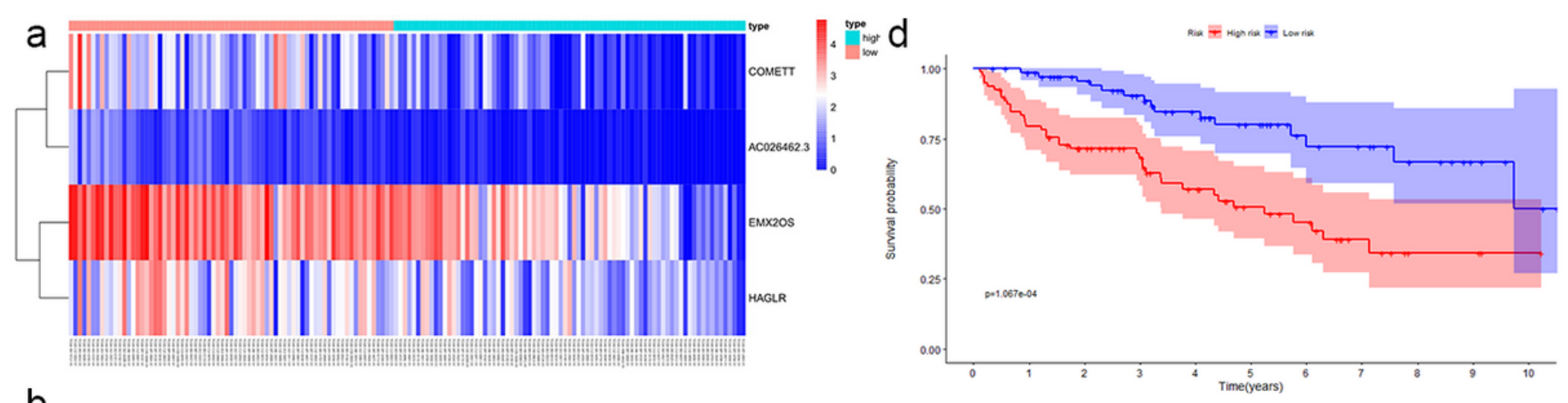

b
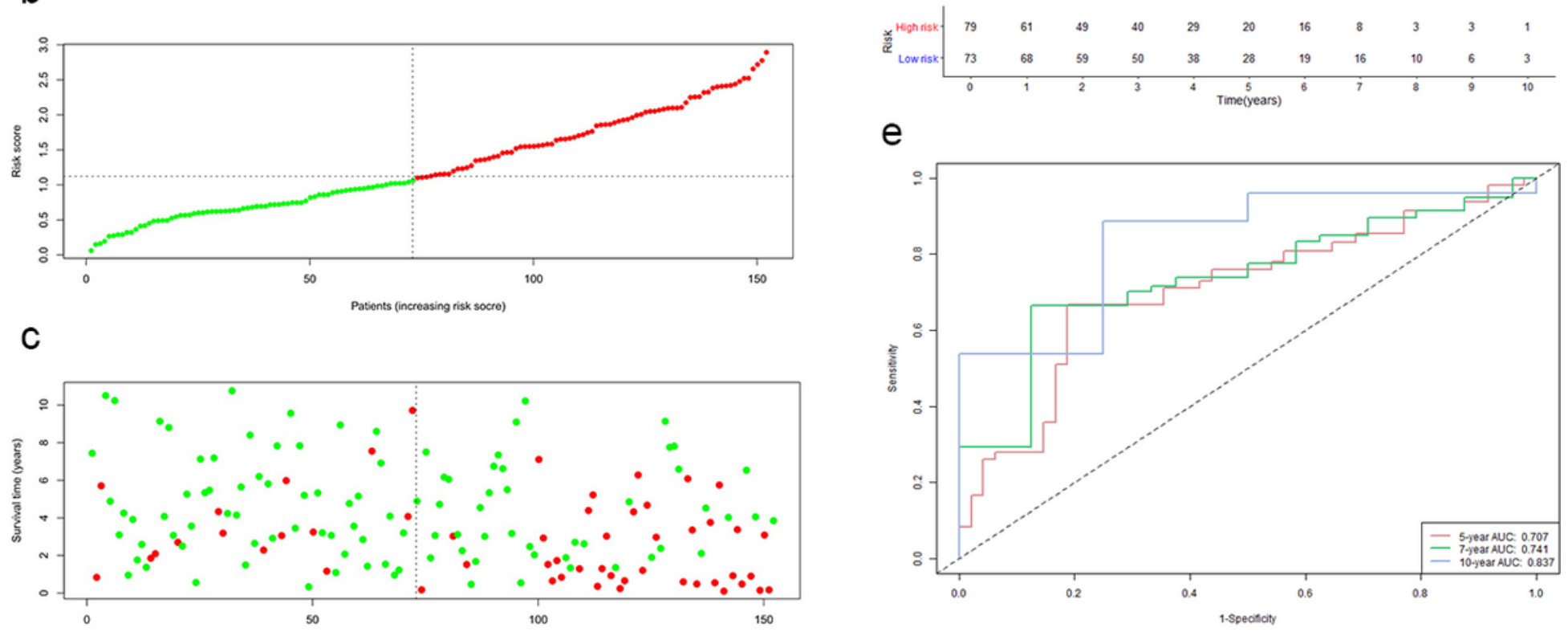

C

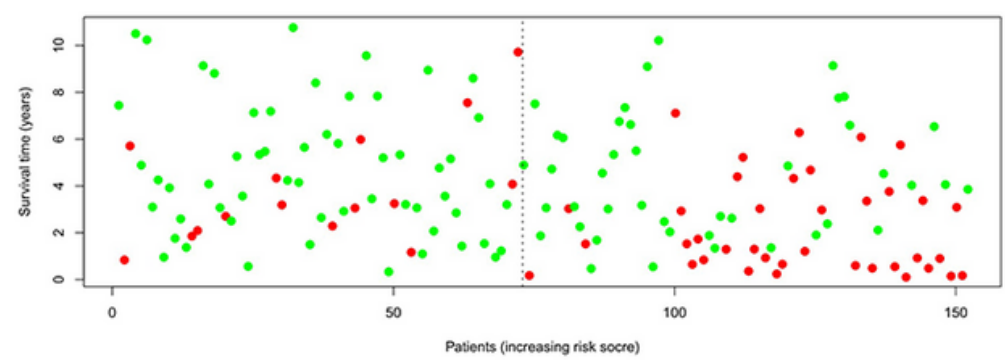

Figure 4

Four hypoxia-related IncRNA signature validated in the testing cohort $(n=152)$. a Expression values of the four signature IncRNAs. The color from red to blue corresponds to the expression of a IncRNA from high to low. The risk score (b) and survival status (c) of each ccRCC patient distributed in low- and highrisk group. d KM survival analysis between the high-risk group and low-risk group. The lower layer demonstrates the number of alive ccRCC patients in each group. e Time-dependent ROC curves of the signature. 

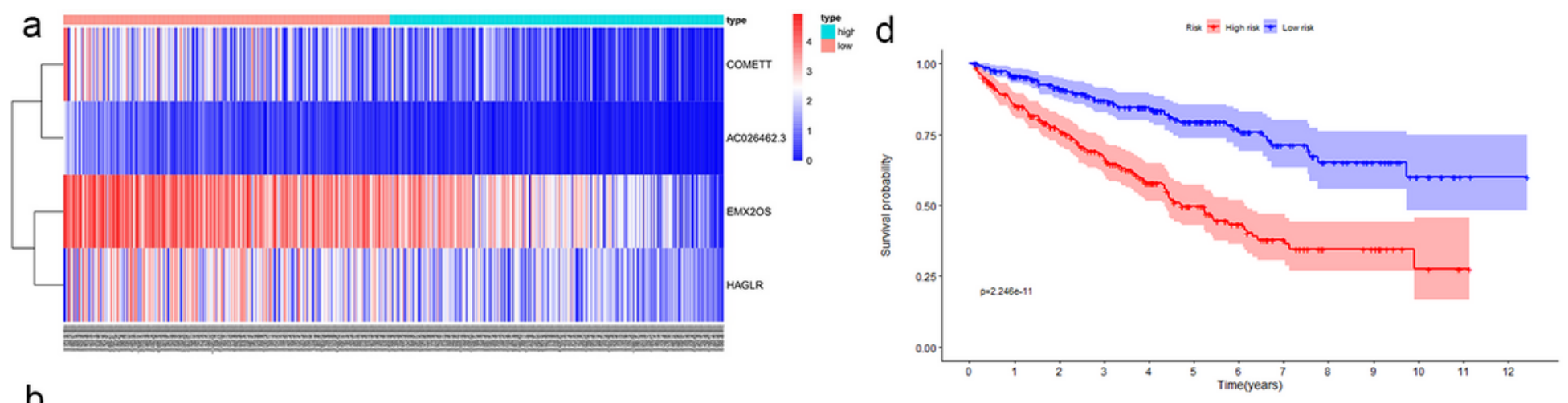

b
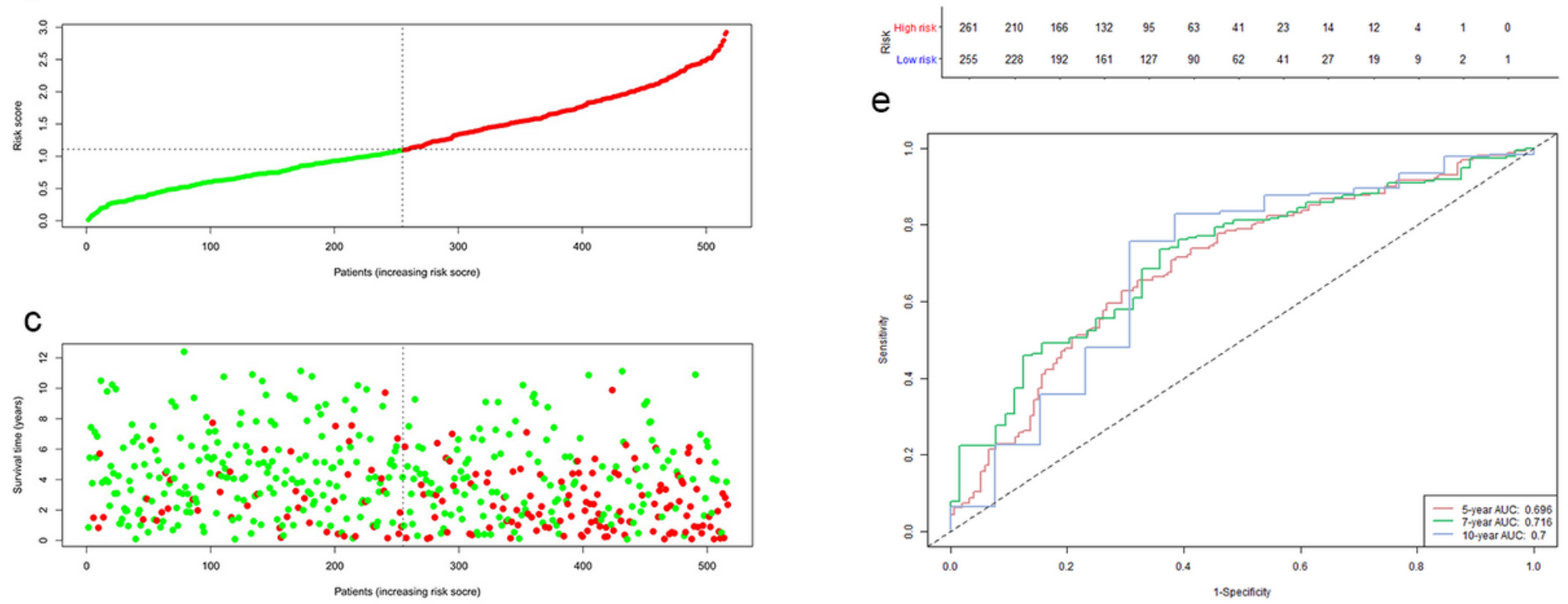

Figure 5

Four hypoxia-related IncRNA signature validated in entire cohort $(n=516)$. a Expression values of the four signature IncRNAs. The color from red to blue corresponds to the expression of a IncRNA from high to low. The risk score (b) and survival status (c) of each ccRCC patient distributed in low- and high-risk group. $d$ KM survival analysis between the high-risk group and low-risk group. The lower layer demonstrates the number of alive ccRCC patients in each group. e Time-dependent ROC curves of the signature. 

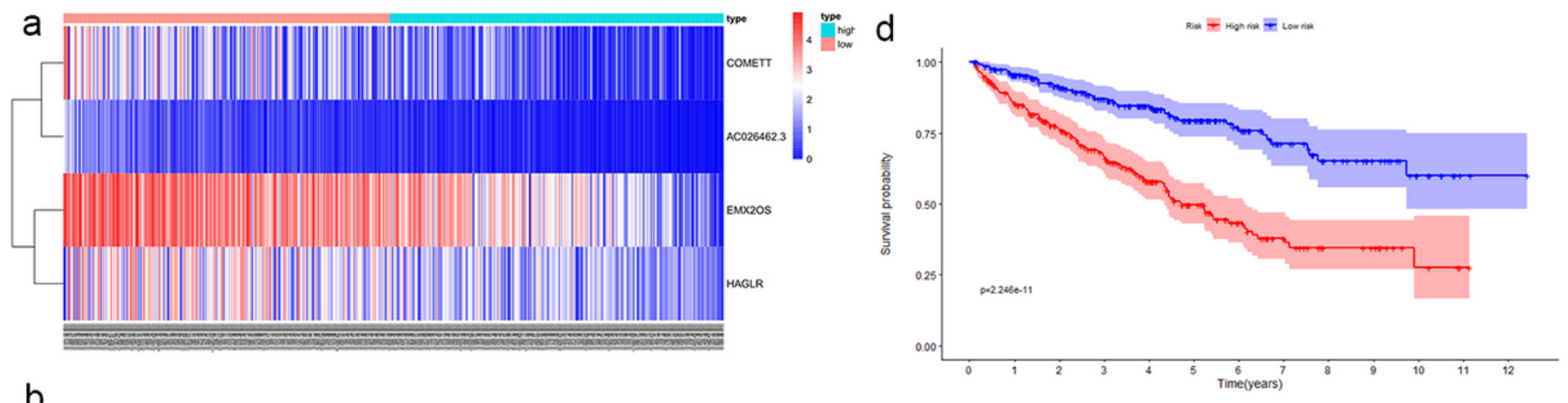

b
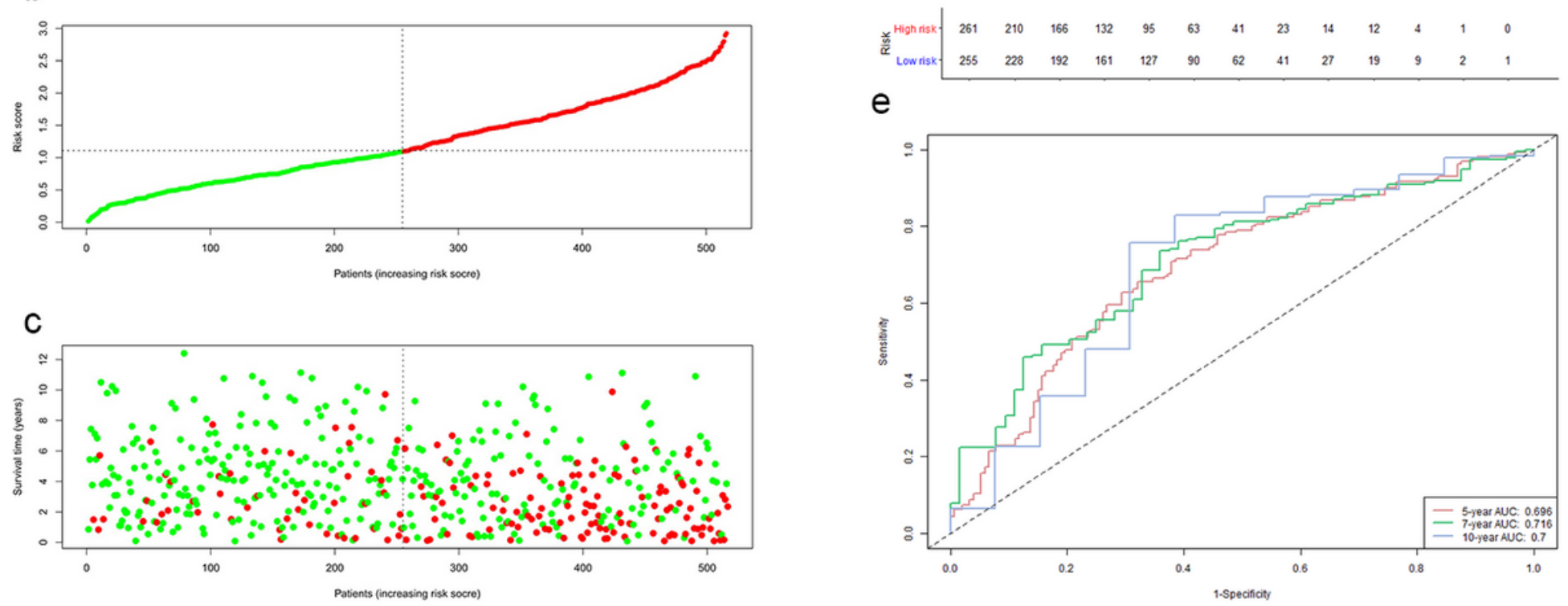

Figure 5

Four hypoxia-related IncRNA signature validated in entire cohort $(n=516)$. a Expression values of the four signature IncRNAs. The color from red to blue corresponds to the expression of a IncRNA from high to low. The risk score (b) and survival status (c) of each ccRCC patient distributed in low- and high-risk group. $d$ KM survival analysis between the high-risk group and low-risk group. The lower layer demonstrates the number of alive ccRCC patients in each group. e Time-dependent ROC curves of the signature. 
a

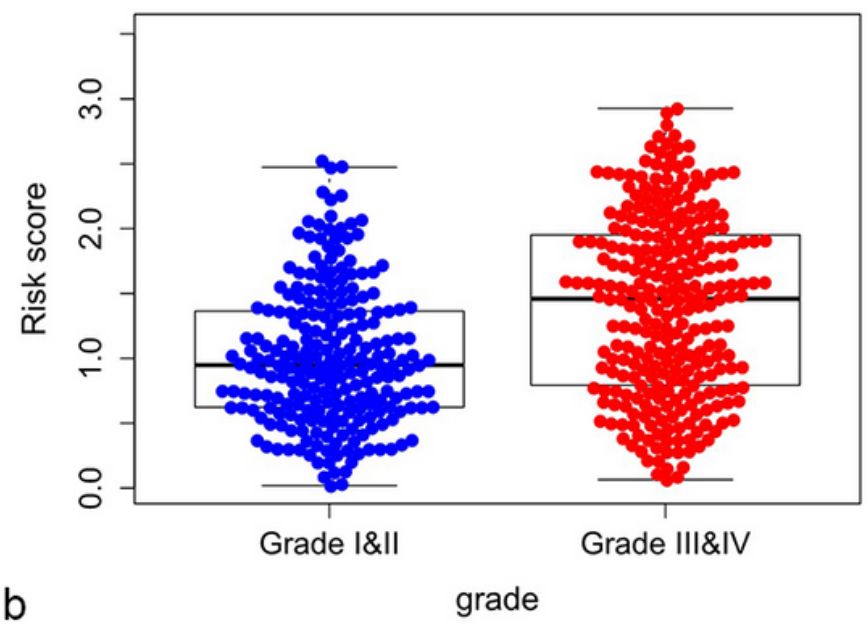

b

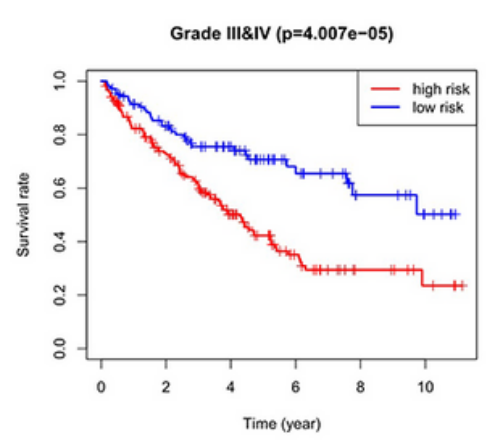

C

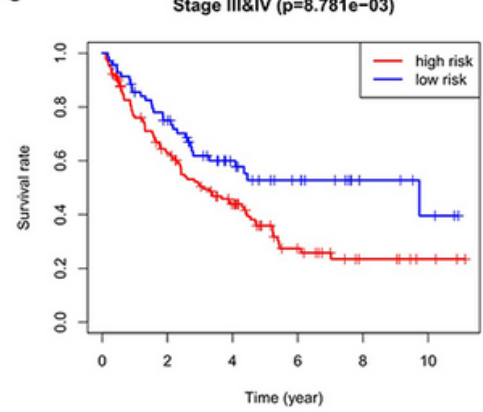

Grade III\&IV

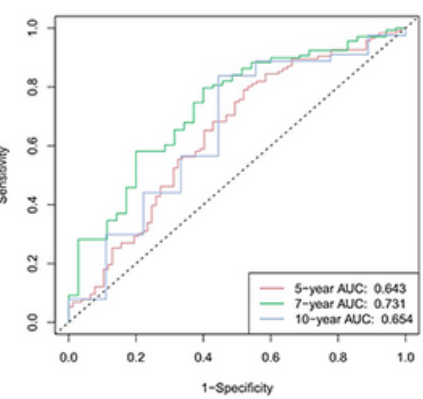

Stage IIIIIV

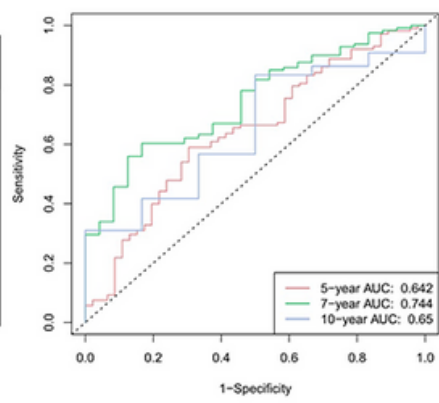

riskScore $(p=2.752 e-13)$
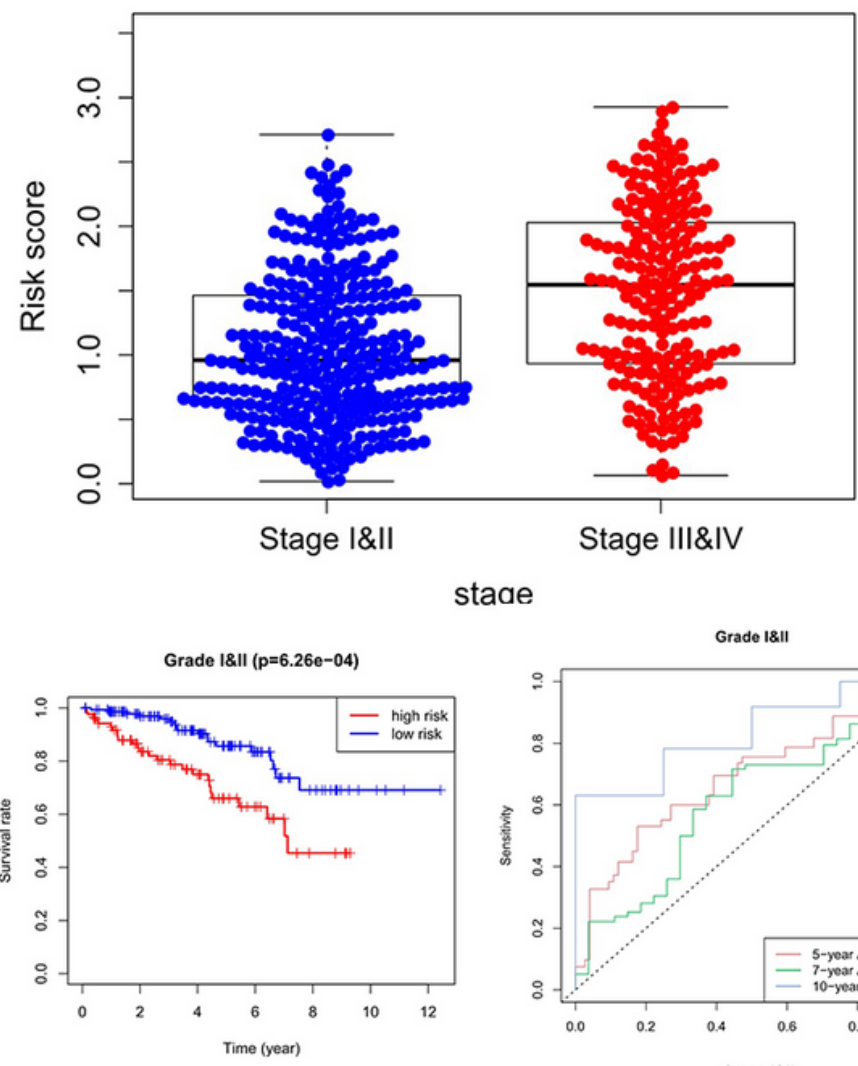

Stage I\&II ( $p=5.308 \mathrm{e}-06)$

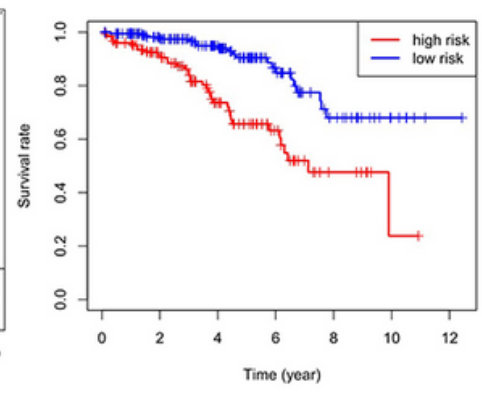

Grade I\&II
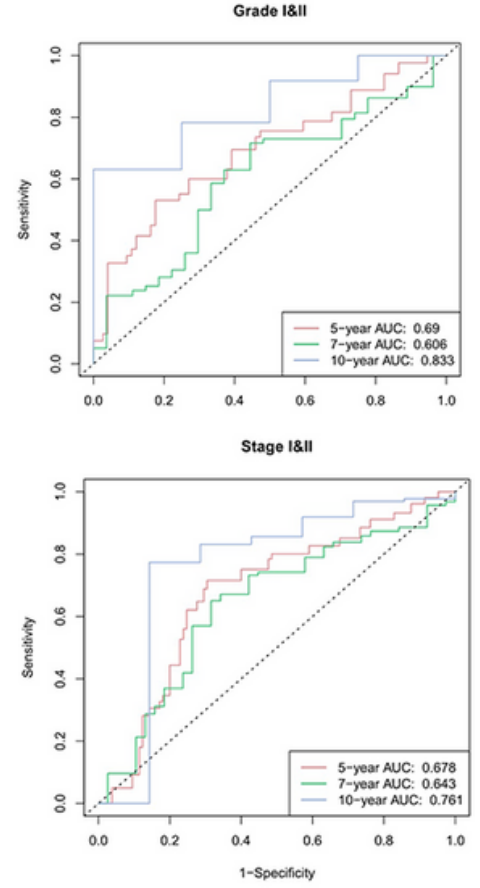

\section{Figure 6}

Relationships between risk score and clinicopathological factors. a Risk score between high grade (grade III\&IV) and low grade (grade I\&II), advanced stage (stage III\&IV), and early stage (stage I\&II). Stratified analysis between the high-risk group and low-risk group in the same grade (b) and stage (c). 
a

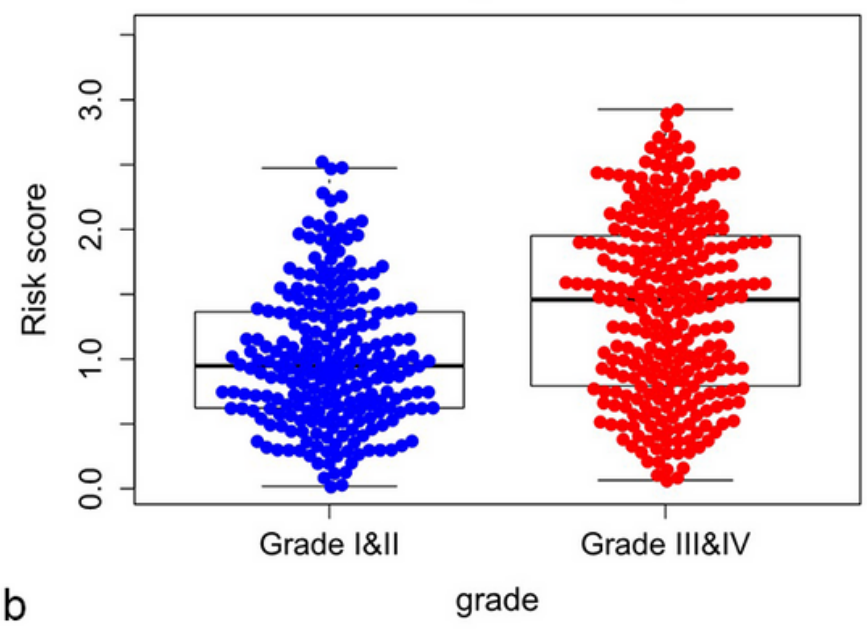

b

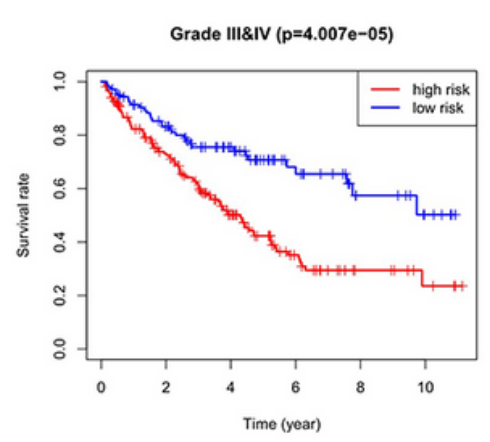

C

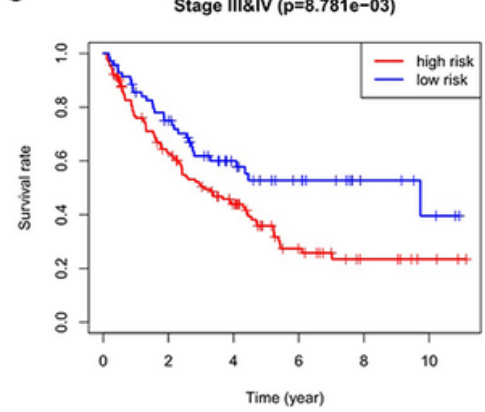

Grade IIIIIV

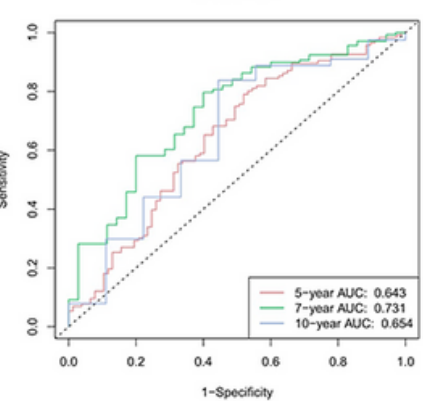

Stage IIIIIV

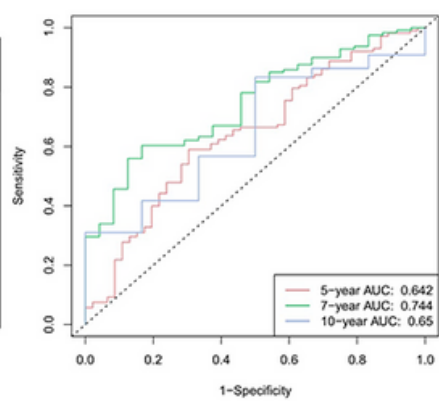

riskScore $(p=2.752 e-13)$

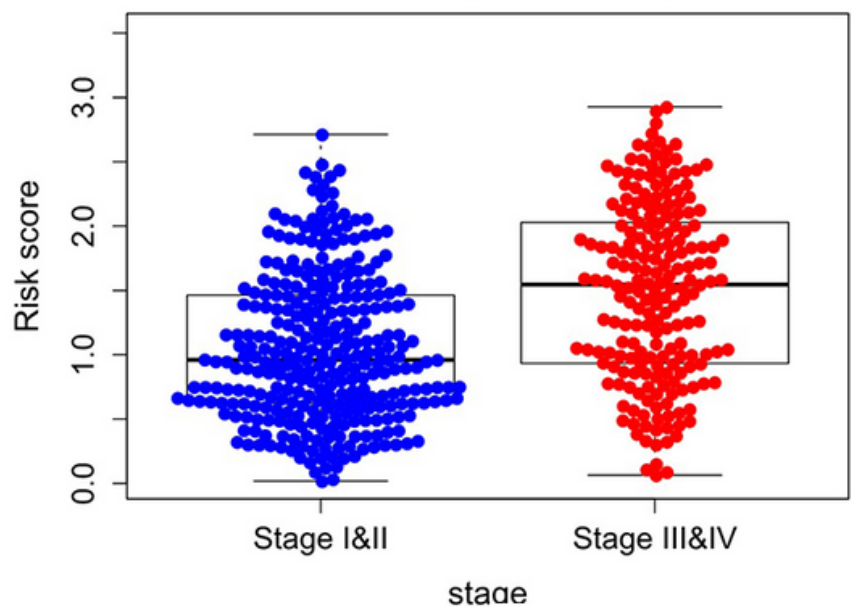

Grade I\&II

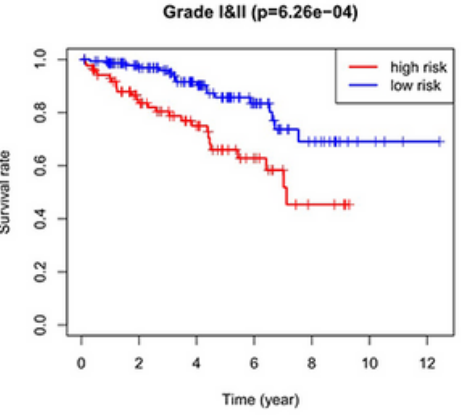

Stage I\&II ( $p=5.308 \mathrm{e}-06)$

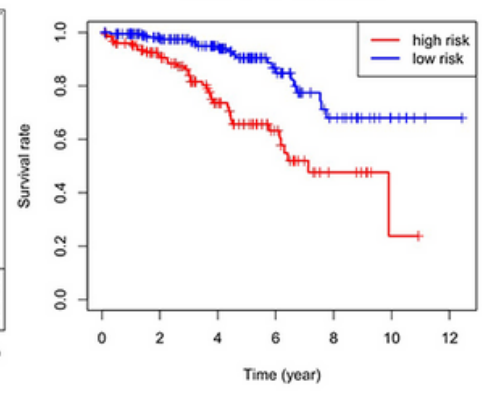

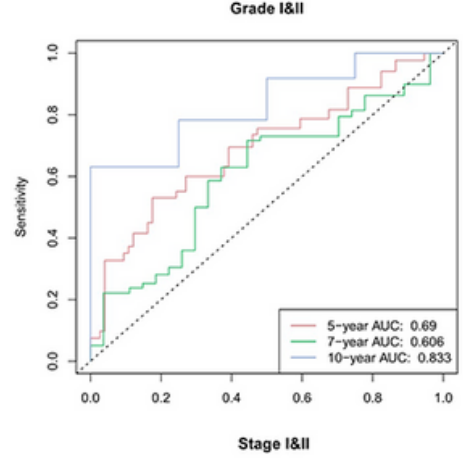

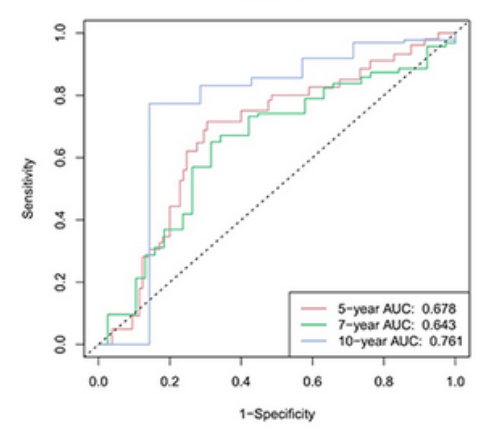

\section{Figure 6}

Relationships between risk score and clinicopathological factors. a Risk score between high grade (grade III\&IV) and low grade (grade I\&II), advanced stage (stage III\&IV), and early stage (stage I\&II). Stratified analysis between the high-risk group and low-risk group in the same grade (b) and stage (c). 

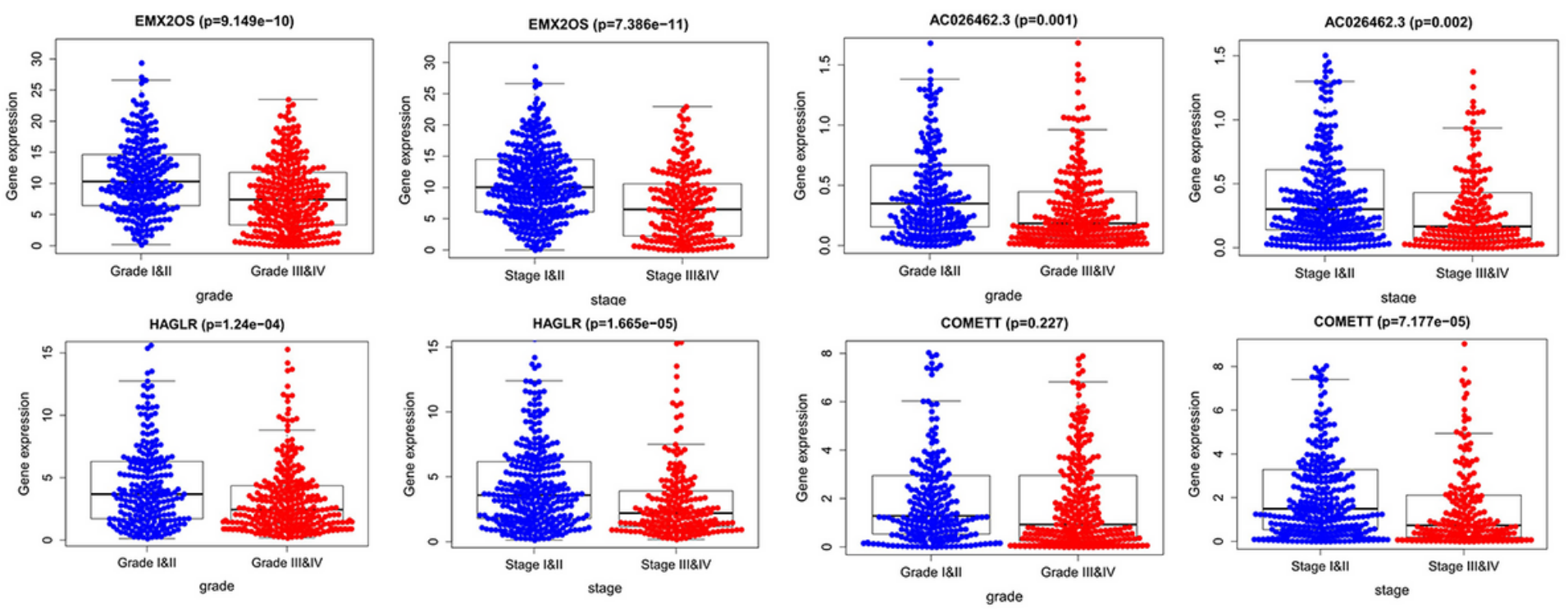

Figure 7

The expression of the four signature IncRNAs between high grade and low grade, advanced stage, and early stage.
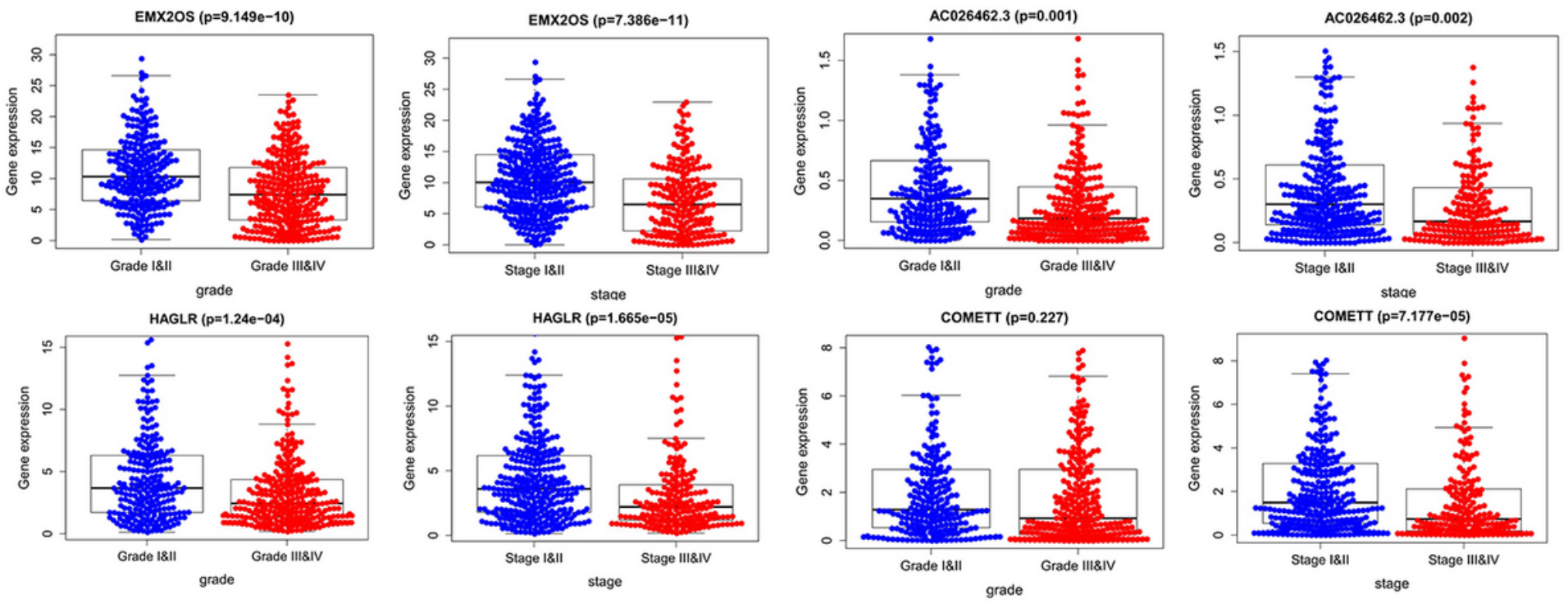

Figure 7

The expression of the four signature IncRNAs between high grade and low grade, advanced stage, and early stage. 

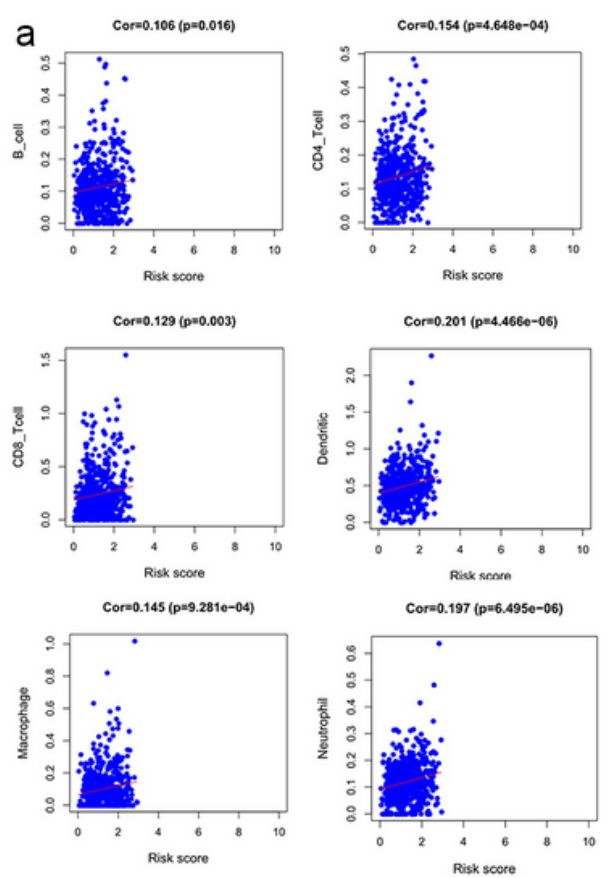

b
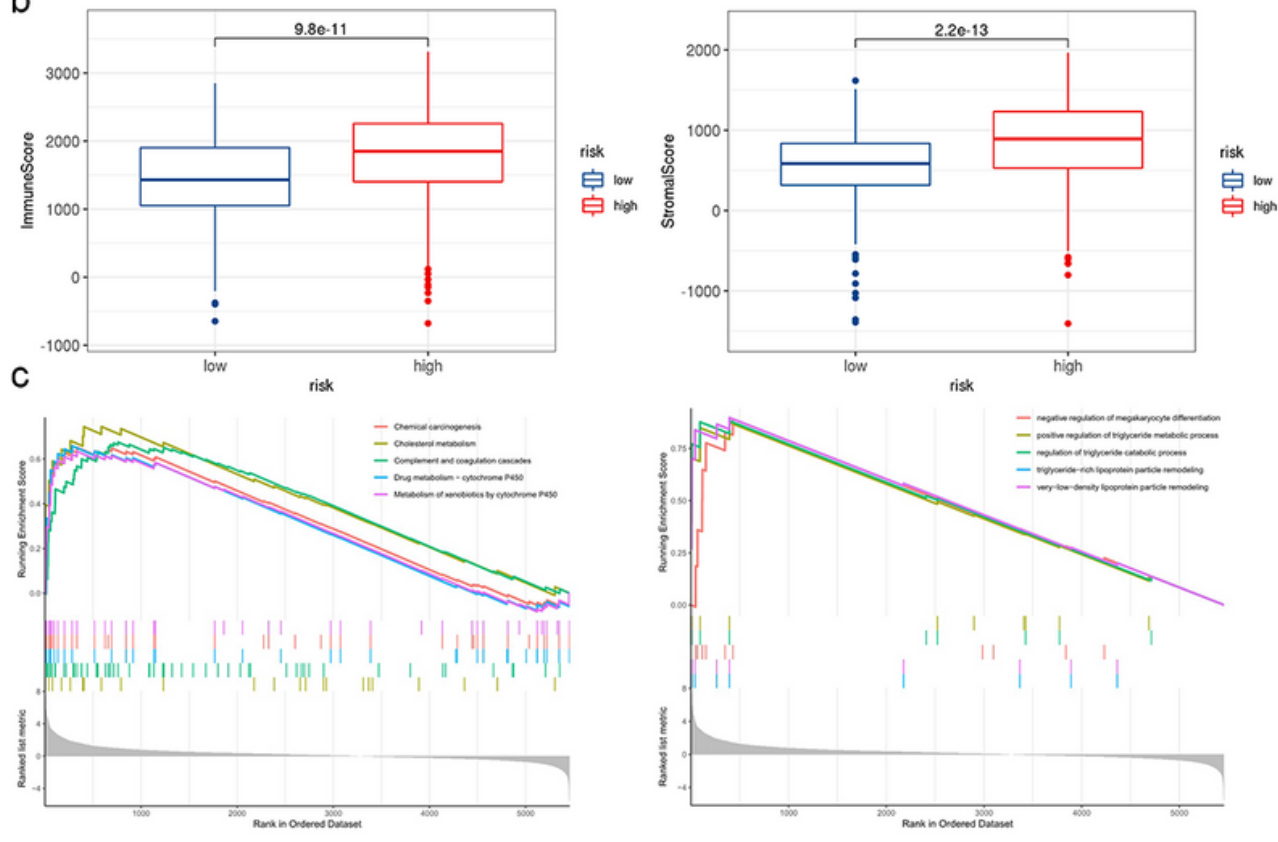

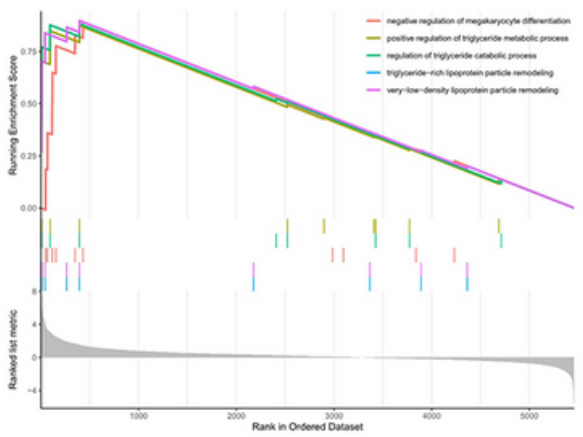

Figure 8

Immune infiltration correlation analysis and GSEA. a Correlation between risk score and immune infiltration abundances in ccRCC of six immune cells obtained from TIMER (https://cistrome.shinyapps.io/timer/). b Relationships between risk score and immune score, and stromal score. c GSEA in TCGA KIRC dataset.
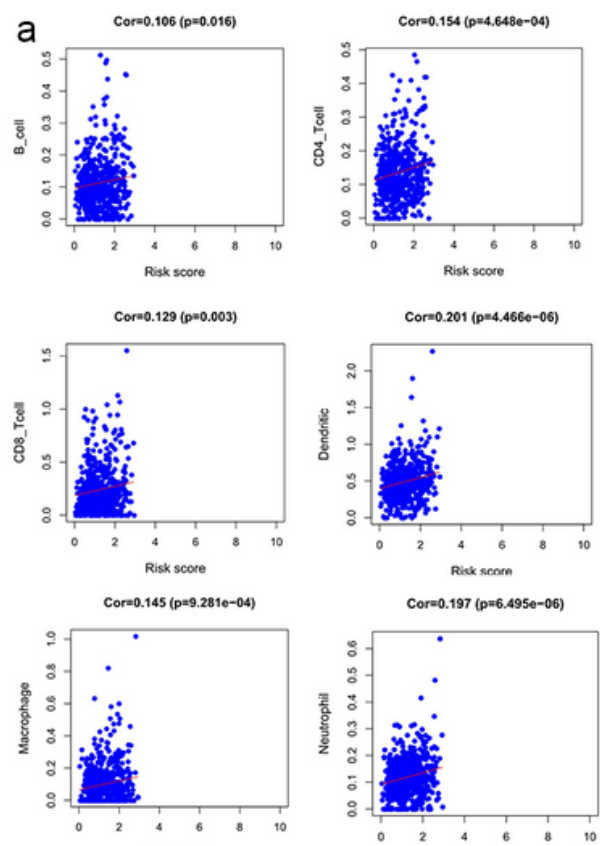

Risk score b
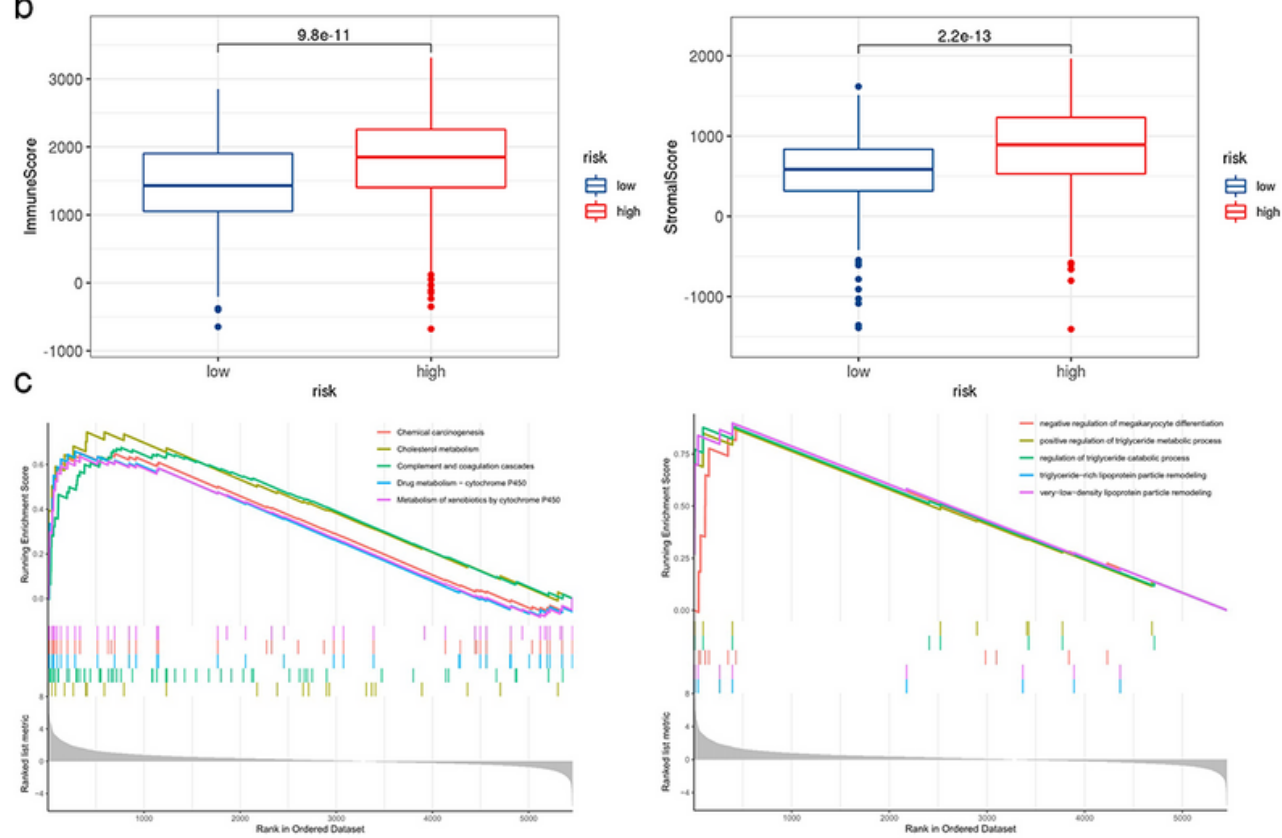

risk

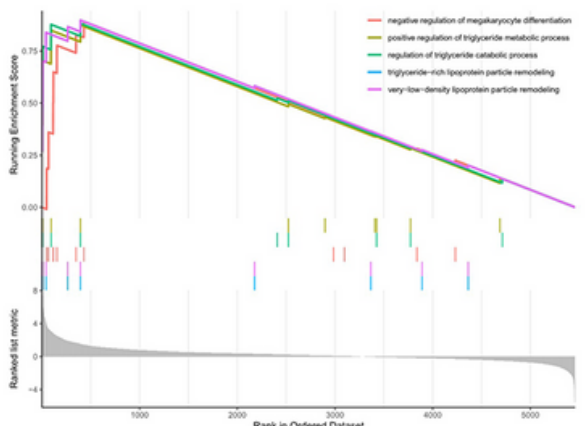

Figure 8

Immune infiltration correlation analysis and GSEA. a Correlation between risk score and immune infiltration abundances in ccRCC of six immune cells obtained from TIMER 
(https://cistrome.shinyapps.io/timer/). b Relationships between risk score and immune score, and stromal score. c GSEA in TCGA KIRC dataset.

a

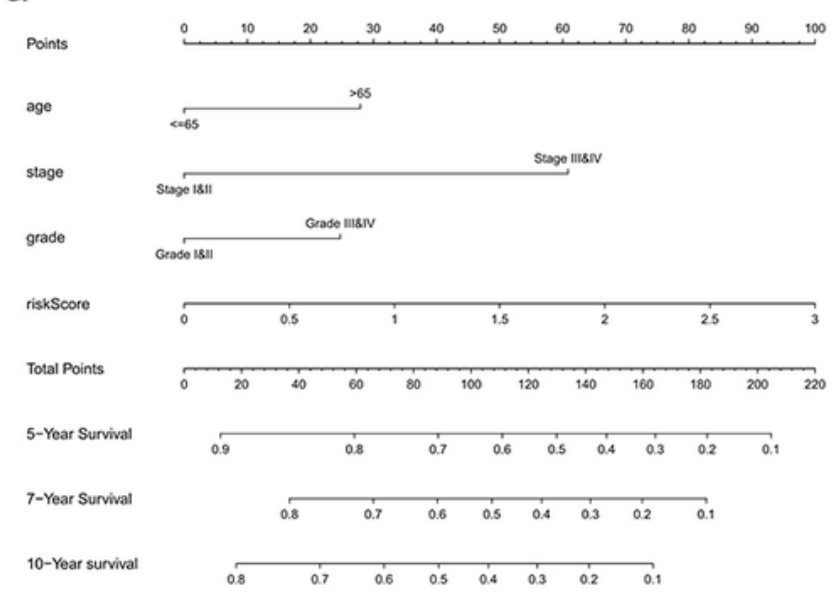

C

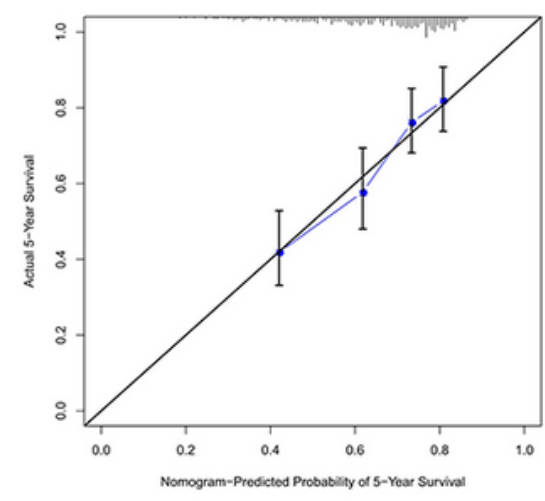

d

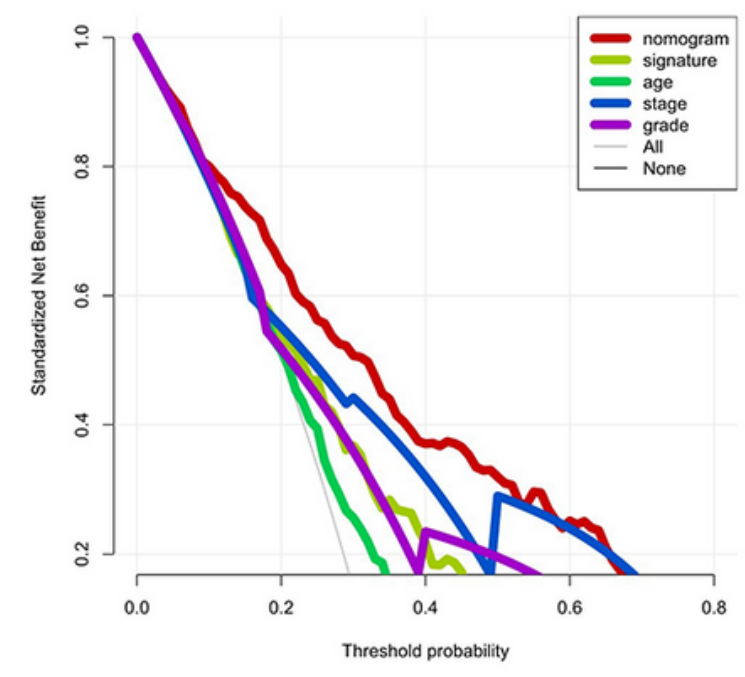

b
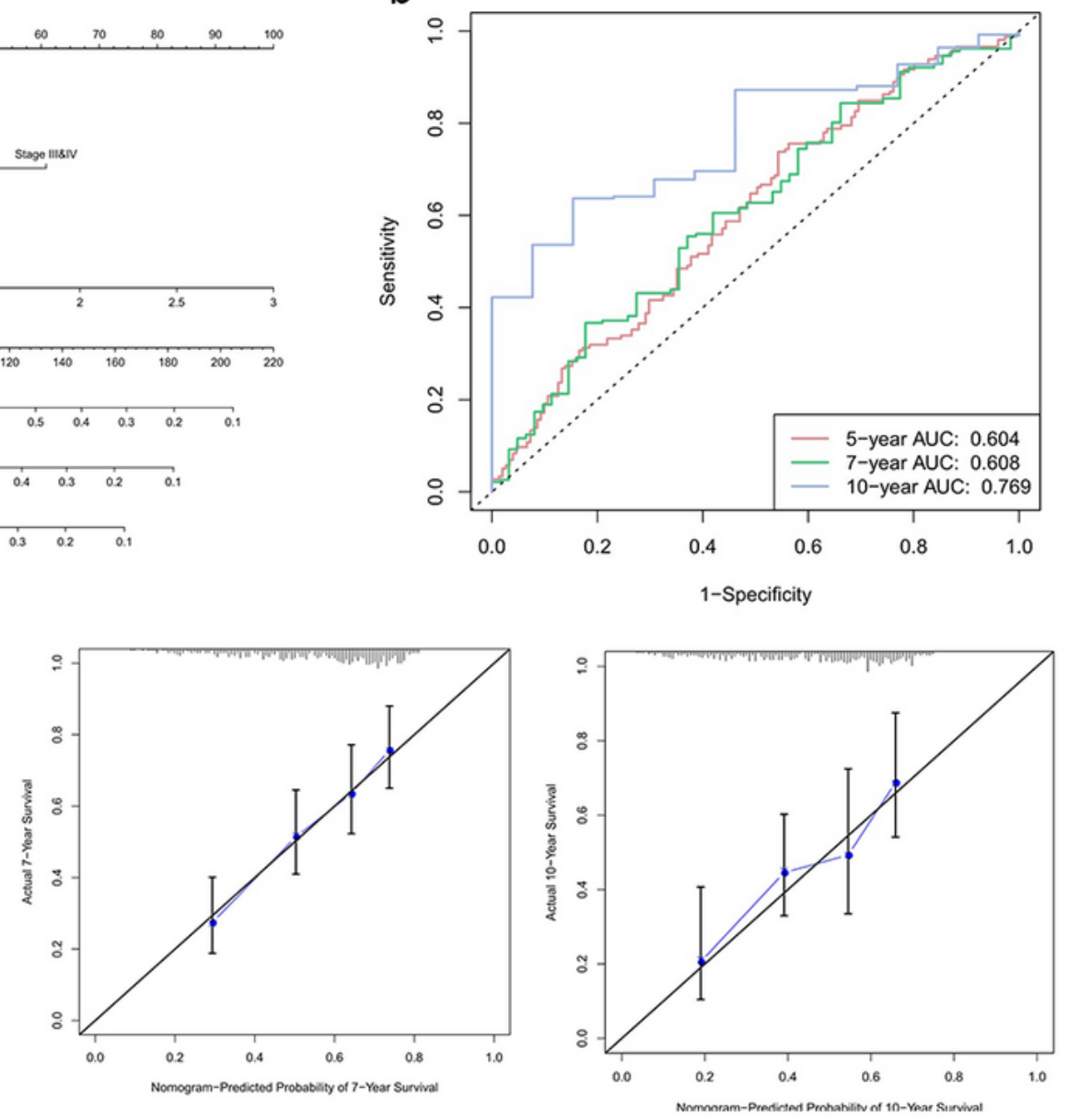

e

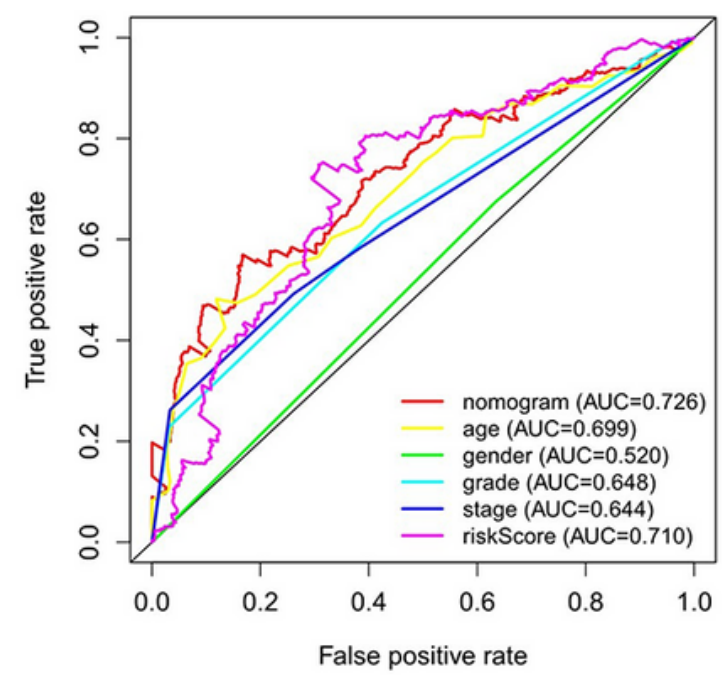

Figure 9

Nomogram construction and performance test. a Nomogram based on risk score, age, stage, and grade. b Time-dependent ROC curves demonstrate the performance of the nomogram in up to 10-year follow-up. C 
Calibration curves of the nomogram for predicting the survival outcomes at 5-, 7- and 10-years. The 45degree line represents the ideal prediction. $d$ Decision curve analyses curve of the nomograms.

a

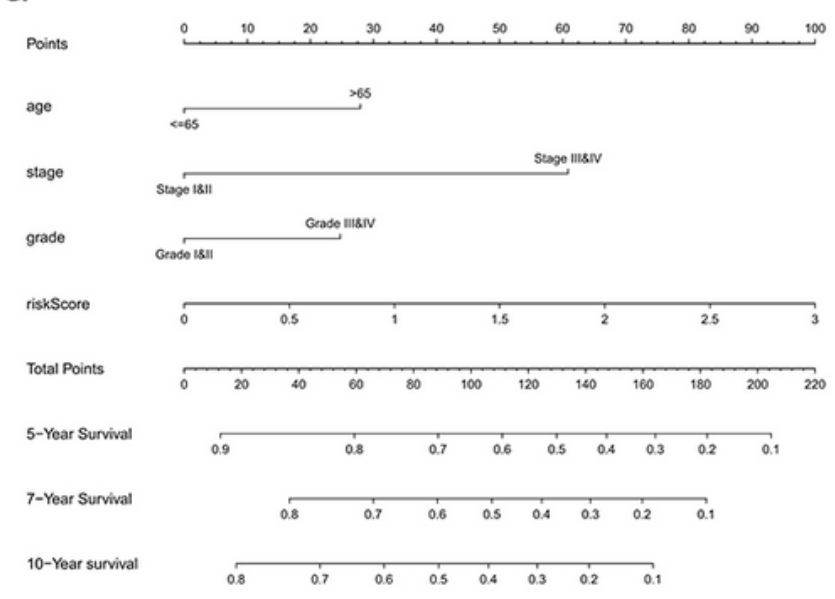

C

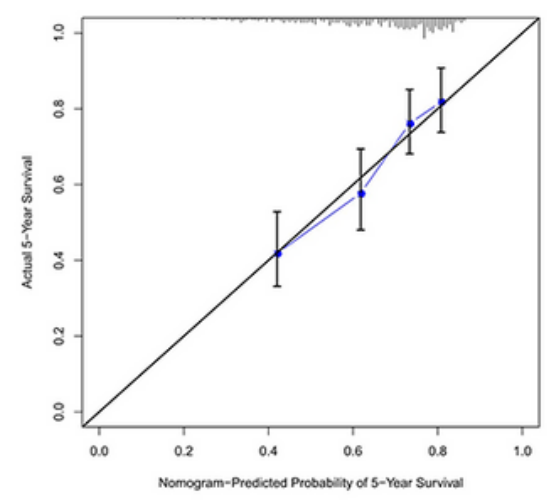

d

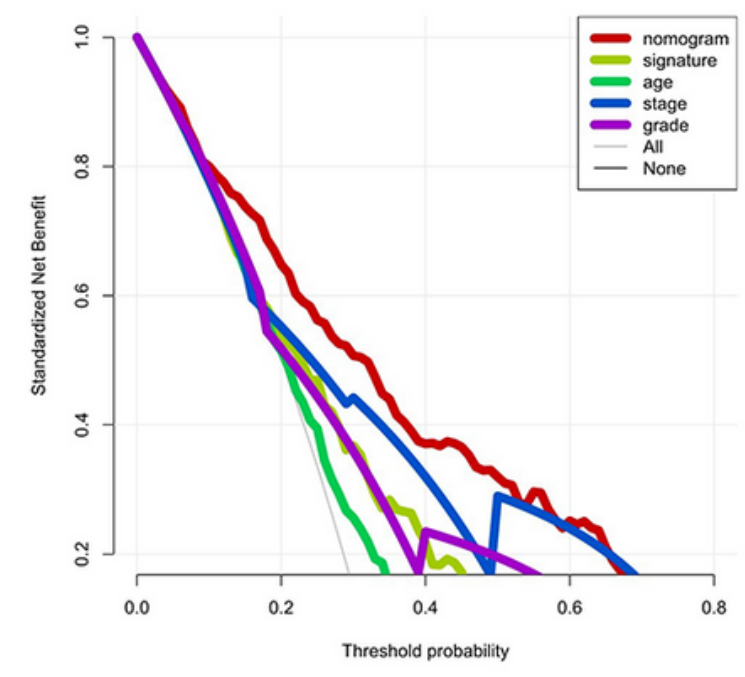

b
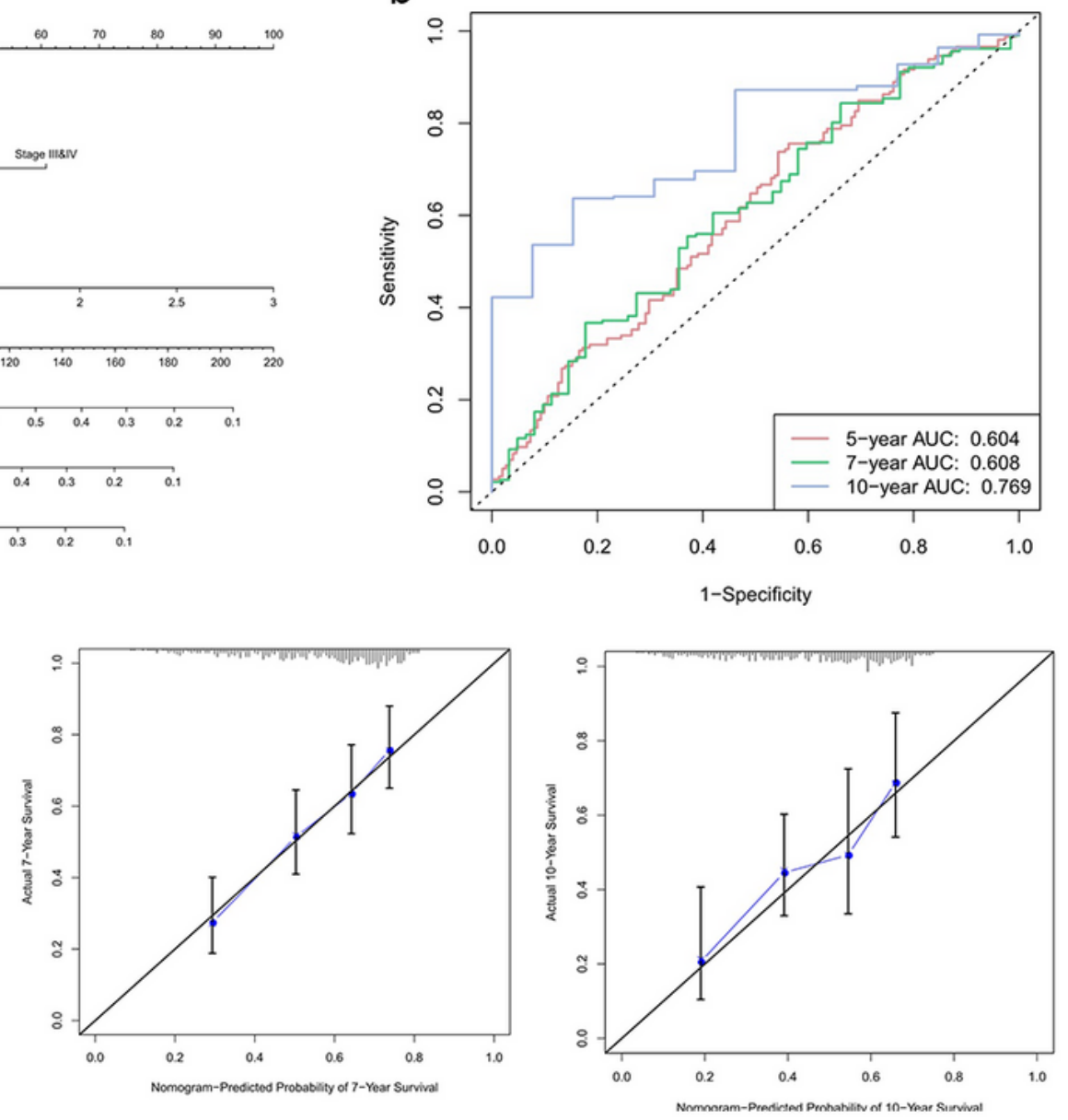

e

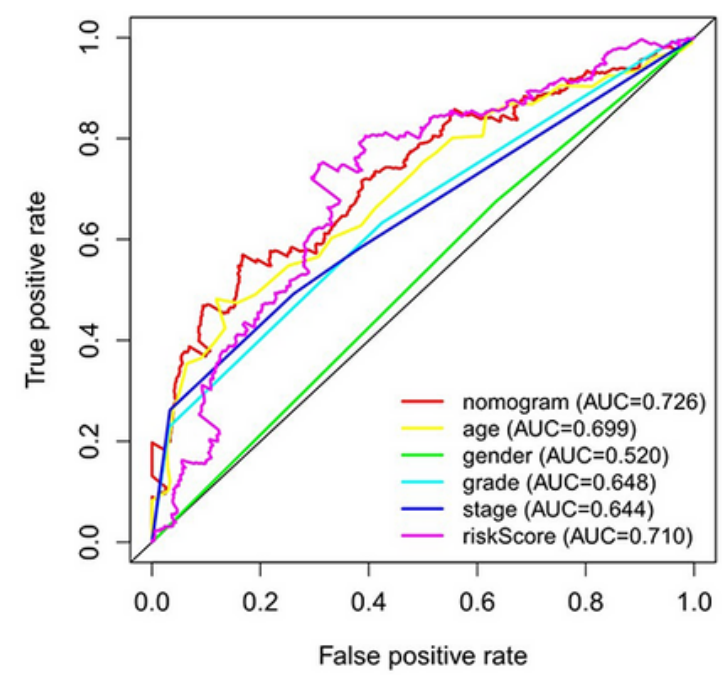

Figure 9

Nomogram construction and performance test. a Nomogram based on risk score, age, stage, and grade. b Time-dependent ROC curves demonstrate the performance of the nomogram in up to 10-year follow-up. C 
Calibration curves of the nomogram for predicting the survival outcomes at 5-, 7- and 10-years. The 45degree line represents the ideal prediction. $d$ Decision curve analyses curve of the nomograms.

\section{Supplementary Files}

This is a list of supplementary files associated with this preprint. Click to download.

- SupplementaryFig.S1.pdf

- SupplementaryFig.S1.pdf 